Andrea Martinangeli

Peter Martinsson

Amrish Patel

\title{
Coordination via Redistribution
}

Max Planck Institute for Tax Law and Public Finance

Working Paper 2017 - 07

November 2017

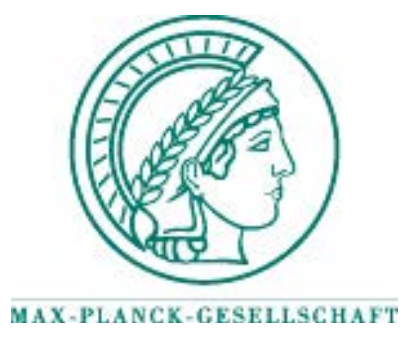

Max Planck Institute for

Tax Law and Public Finance

Department of Business and Tax Law

Department of Public Economics

http:/ / www.tax.mpg.de 
Working papers of the Max Planck Institute for Tax Law and Public Finance Research Paper Series serve to disseminate the research results of work in progress prior to publication to encourage the exchange of ideas and academic debate. Inclusion of a paper in the Research Paper Series does not constitute publication and should not limit publication in any other venue. The preprints published by the Max Planck Institute for Tax Law and Public Finance represent the views of the respective author(s) and not of the Institute as a whole. Copyright remains with the author(s).

Max Planck Institute for Tax Law and Public Finance

Marstallplatz 1

D-80539 Munich

Tel: $\quad+498924246-0$

Fax: $\quad+498924246-501$

E-mail: ssrn@tax.mpg.de

http://www.tax.mpg.de 


\title{
Coordination via Redistribution*
}

\author{
Andrea Martinangeli ${ }^{\dagger}$ Peter Martinsson ${ }^{\ddagger}$ and Amrish Patel ${ }^{\S}$
}

November 22, 2017

\begin{abstract}
Can prior voluntary redistribution improve coordination? We theoretically show that distributive preferences, forward induction and signalling all imply that it can. We then experimentally test our predictions by allowing subjects to redistribute part of their endowment before playing a battle of the sexes game. To identify whether the redistribution option increases coordination, and why, we also run experiments with no redistribution and forced redistribution. Our results show that the redistribution option does indeed significantly increase coordination. Disentangling the reasons why, we find that behaviour is most consistent with distributive preferences and one-step of forward induction (rather than signalling or two-steps of forward induction).
\end{abstract}

Keywords: Coordination, redistribution, experiment, distributive preferences, forward induction, signalling altruism.

JEL codes: C72, D02

\section{Introduction}

Coordination is an important ingredient for economic success in many situations, from efficient teamwork, to the adoption of technology, to the organisation of social activities.

\footnotetext{
${ }^{*}$ We thank Oana Borcan, David Cooper, Martin Dufwenberg, Andrea Isoni, Sverker Jagers, Olof Johansson Stenman, Martin Kocher, Thomas Markussen, Katarina Nordblom, Anders Poulsen, Rupert Sausgruber, Johan Stennek, Bob Sugden and several seminar audiences for useful comments or discussions. We gratefully acknowledge the assistance of Martin Kocher, David Schindler and the MELESSA laboratory staff in organising the experiment. This work received financial support from FORMAS via the COMMONs Research Program.

${ }^{\dagger}$ Max Planck Institute for Tax and Public Finance; andrea.martinangeli@tax.mpg.de

${ }^{\ddagger}$ University of Gothenburg; Linköping University; peter.martinsson@economics.gu.se

${ }^{\S}$ University of East Anglia; amrish.patel@uea.ac.uk
} 
Despite the often large mutual gains of coordination, the difficulty of anticipating others' choices makes coordination challenging, particularly when there is no payoff or risk dominant equilibrium for equilibrium selection (e.g. Harsanyi and Selten 1988). ${ }^{1}$

A central question in the literature on coordination is which coordination devices can improve equilibrium selection and hence coordination rates. Previous empirical results have shown that deductive criteria for equilibrium selection such as focal points (Schelling 1960, Crawford et al. 2008), communication (Cooper et al. 1989) and abstaining from an outside option (Cooper et al. 1993) can serve as successful coordination devices, helping individuals reason how to coordinate. Many of these devices share the feature that they need to be added to the coordination situation, i.e. an intervention in the choice architecture is needed.

This paper departs from considering a specific coordination problem in isolation and instead investigates the role of past behaviour as a potential coordination device rather than imposed devices. Note that our focus is on past behaviour in other situations, not learning and adaptation within the coordination situation itself (e.g. Van Huyck et al. 1997).

Past behaviour in other situations can take many forms. One important class of behaviours may be thought of as voluntary redistribution in one way or the other. Suppose that you are in a coordination situation with individual A. You learn that before the coordination situation, she made a large donation to a local charity or spent a lot of time training junior employees at work. Might such voluntary redistribution and knowledge of it provide deductive criteria for equilibrium selection and thus help coordination?

A simple intuition as to why it might is as follows: Individual A was generous yesterday (i.e. redistributed part of her income to someone else); today you coordinate on the equilibrium that she prefers (i.e. increase her income (or utility)) in order to minimise payoff (or utility) differences.

To study how past voluntary redistribution affects coordination, we examine coordination in two-stage coordination games where a player may have the option to redistribute part of her endowment to a third-party before the coordination game.

The basic coordination problem is captured using the classic "battle of the sexes" game. It represents situations where players have a conflict of interest over which outcome to coordinate on. Figure 1 exemplifies the game. In essence, it is an asymmetric $2 \mathrm{x} 2$ coordination game with two players who simultaneously and independently choose between two strategies.

\footnotetext{
${ }^{1}$ An equilibrium is risk dominant if the product of the payoff reduction for each player from unilateral deviations is higher than other equilibria.
} 
Figure 1: The 'battle of the sexes' game

\begin{tabular}{cccc} 
& & \multicolumn{2}{c}{ Player B } \\
& & $\underline{\text { High }}$ & $\underline{\text { Low }}$ \\
\cline { 3 - 4 } Player A & High & 0,0 & 6,3 \\
& Low & 3,6 & 0,0 \\
\hline
\end{tabular}

Notes: Coordinated outcomes shaded. Player B's action choices will be underlined henceforth.

The game has two pure strategy Nash Equilibria (High-Low and Low-High). While players prefer to coordinate on these profiles than not do so, each player prefers a different Nash Equilibrium, hence the conflict of interest. Unsurprisingly, coordination rates in lab implementations of the battle of the sexes game are low (e.g. $41 \%$ in Cooper et al. 1989), close to the $50 \%$ coordination predicted if individuals were to randomise uniformly.

To appropriately incorporate voluntary redistribution, it is worth returning to our two examples. Both donations to charity and training a junior co-worker involve a transfer from a relatively better off member of society (or one with the ability to become so) to a relatively worse off member. Given this, our game involves player A having the option to transfer payoff units to a less well-off third-party (player C), before A and B play the battle of the sexes game. We label this the voluntary transfer game.

To understand why the option to voluntarily redistribute may help coordination, recall the game in Figure 1 and suppose that A can redistribute three payoff units to a less welloff third-party. If A chooses to redistribute, while there are still two Nash Equilibria, one of them (High-Low) will now give a more equal payoff distribution. The more equitable payoff distribution is attractive for individuals who have distributive preferences and dislike payoff differences (e.g. Fehr and Schmidt 1999; Bolton and Ockenfels 2000; Charness and Rabin $2002^{2}$ ), thus potentially solving the coordination problem.

This is not the only reason why a redistribution option can help coordination. A's choice to transfer or not can potentially signal her intended choice in the coordination game to B, thereby facilitating coordination. This is the idea of forward induction (Kohlberg and Mertens 1986).

To illustrate one-step of forward induction reasoning, suppose that A chooses to transfer three payoff units to $\mathrm{C}$ before the game in Figure 1 is played. What can $\mathrm{B}$ learn about A's intended choice in the coordination game? B would reason that A cannot intend to play Low as A's payoff would be weakly higher if she had chosen not to transfer, then played Low. Thus it must be that A intends to play High, in which case B should play Low. Hence coordination is possible. The logic is identical to that in money-burning games (van Damme 1989; Ben-Porath and Dekel 1992).

Redistribution may seem to make coordination trivial, however this is not the case.

\footnotetext{
${ }^{2}$ For a general overview see Fehr and Schmidt 2006
} 
For example, suppose that A uses her transfer choice to signal her privately known degree of altruism (e.g. Levine 1998, Benabou and Tirole 2006). Transferring would then signal to $\mathrm{B}$ that $\mathrm{A}$ is altruistic and thus that they should coordinate on the profile which gives $\mathrm{B}$ a higher payoff, Low-High. Notice that these are precisely the opposite action choices to those predicted by one-step of forward induction and inequity-aversion.

Formalising the above intuitions, we theoretically demonstrate (in the next secion) how distributive preferences, forward induction and signalling can all imply coordination when A has the option to redistribute before the coordination game.

Identifying the effect of voluntary redistribution on coordination in naturally occurring data is challenging due to the many confounding factors and endogeneity (for a discussion of the pros and cons of different data collection methods see Falk and Heckman 2009). We thus run a controlled laboratory experiment to identify whether voluntary redistribution increases coordination, and if so, why.

Some of the theories we study make different predictions in our game (e.g. signalling altruism versus inequity-aversion) allowing us to distinguish between them empirically. To distinguish between theories whose predictions overlap in the voluntary transfer game, we also implement an additional game, the mandatory transfer game. In this game, A is forced to transfer to $\mathrm{C}$ before the coordination game in Figure 1 is played. That $\mathrm{A}$ makes no choice implies that neither forward induction nor signalling can explain coordination. Distributive preferences can still imply coordination in this game. By comparing coordination rates in the standard battle of the sexes to the mandatory transfer game, we isolate the effect of distributive preferences. By comparing coordination rates between the mandatory transfer game and the voluntary transfer game conditional on a transfer being made, we isolate the effect of signalling and forward induction.

We find that aggregate coordination rates are significantly higher in the mandatory and voluntary transfer games (66.4\% and $62.7 \%$ respectively) than the standard battle of the sexes $(47.7 \%)$. That aggregate coordination rates are not significantly different across the two transfer games would suggest that inequity aversion, not forward induction and signalling explain successful coordination. However, a closer look at behaviour suggests otherwise. Conditional on A choosing to transfer, the coordination rate in the voluntary transfer game is $82.0 \%$, significantly higher than that in the mandatory transfer game. A transfer should be equally effective regardless of whether it is voluntary or mandatory according to inequity-aversion, whereas forward induction and signalling work only when the transfer is voluntary. Since the choices observed in the coordination game are inconsistent with those predicted by signalling altruism, we conclude that most behaviour is explained by inequity-aversion and one-step of forward induction.

Our work contributes to several literatures. First we add to a literature studying how ex-ante transfers can lead to more efficient outcomes in general games (Jackson and Wilkie 2005) and in social dilemmas (Charness et al. 2007). We provide the first systematic study of whether voluntary ex-ante transfers affect coordination and explain why they do so. 
Second, we add to a large experimental literature on coordination games. Our voluntary transfer game is theoretically similar to a money burning game (van Damme 1989; Ben-Porath and Dekel 1992), in that forward induction predicts the first mover not sacrificing money and then players coordinating on her preferred choice. In our voluntary transfer game, the analog would be A not transferring and then players coordinating on her preferred choice. Unlike experiments on money burning (e.g. Huck and Müller 2005), the prediction of forward induction is not consistent with our data, since coordination only occurs if A transfers in our game. Our data is however consistent with limited forward induction, i.e. one-step of forward induction reasoning. The difference suggests that redistribution is not the same as money-burning when it comes to their effect on coordination.

Our data is also consistent with recent literature arguing that social preferences may be beneficial for coordination (e.g. Chen and Chen 2011; Dufwenberg and Patel 2017). The opposing forces of social preferences and forward induction have been noted in a coordination game with an outside option (Shahriar 2013), but not for games involving payoff sacrifices as in ours.

We proceed as follows. Section 2 presents and theoretically analyses our experimental games (2.1), derives hypotheses (2.2) and details experimental procedures (2.3). Section 3 describes results on whether a voluntary redistribution option increases coordination (3.1) and if so, why it does so (3.2). Section 4 critically reflects on our analysis and concludes.

\section{Experimental framework and hypotheses}

In this section we introduce our experimental framework. We first present our three conditions, focusing on developing theoretical predictions that follow from different assumptions on players' motivations and reasoning processes. These predictions imply clear testable hypotheses. Finally, we describe our experimental procedures.

Our experimental design consists of three conditions: No Transfer ${ }^{3}$, Voluntary Transfer and Mandatory Transfer. All three conditions involve a group of three players (A, B and C) and each player has an endowment of 12 payoff units. Each condition proceeds in two stages. In the first stage, there may or may not be a fixed transfer of three payoff units from A to C. In all conditions, the second stage is identical to the game in Figure 1. We compare second stage coordination rates across the three conditions. The conditions differ only in the first stage: in the No Transfer Condition, A cannot transfer; in the Mandatory Transfer Condition, A is forced to transfer; in the Voluntary Transfer Condition, A can voluntarily choose whether to transfer or not.

The game forms played in our conditions are depicted in Figures 2-4 that follow. ${ }^{4}$ The

\footnotetext{
${ }^{3}$ We use the words "redistribution" and "transfer" interchangeably. Redistribution is primarily used in motivation and discussions sections; transfer in more analytical sections.

${ }^{4}$ Note that endowments are excluded from the payoffs in Figures 2-4. Final monetary payoffs are
} 
game played in the No Transfer Condition is equivalent to a standard battle of the sexes (Figure 1) other than the presence of passive $\mathrm{C}$ and that $\mathrm{A}$ does not transfer money to $\mathrm{C}$ in a prior stage. If $\mathrm{A}$ and $\mathrm{B}$ choose the same action (High-High or Low-Low) in the second stage, then both receive a payoff of zero. If they coordinate on different actions (High-Low or Low- $\underline{\text { High }}$ ), then the one who played high receives six and the other receives three.

Figure 2: The No Transfer Condition

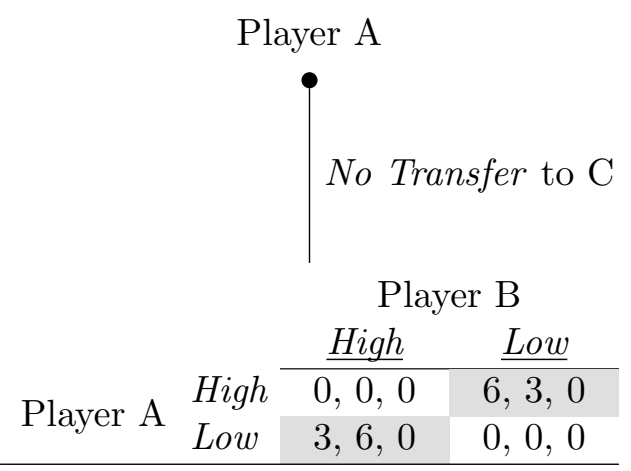

Notes: This figure describes the game form played in the No Transfer Condition, the No Transfer Game, $\Gamma_{N}$. Payoffs excluding endowments are presented as (payoff A, payoff B, payoff C). Coordinated outcomes are shaded.

In the Voluntary Transfer Game, $\Gamma_{V}$, if A chooses No Transfer the ensuing subgame is identical to the No Transfer Game; if A chooses Transfer, three payoff units are transferred from A to C, then A and B play the same battle of the sexes as in the second stage of the No Transfer Game.

To facilitate understanding and see how similar the different conditions are, Figure 3 presents the Voluntary Transfer Game with final payoffs. In the experiment, subjects were not presented with final payoffs, but stage payoffs. That is, regardless of what A chose, in the second stage subjects were presented with identical choices and payoff consequences as the No Transfer Condition.

obtained by adding 12 to the payoffs presented. The theoretical predictions that follow are qualitatively unaffected by this scaling. 
Figure 3: The Voluntary Transfer Condition

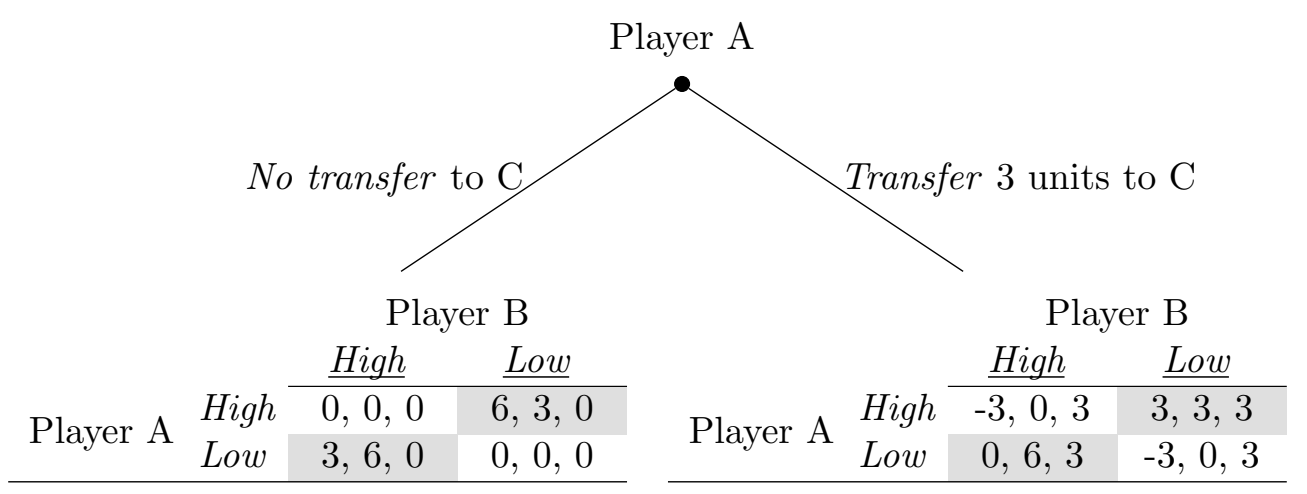

Notes: This figure describes the game form played in the Voluntary Transfer Condition, the Voluntary Transfer Game, $\Gamma_{V}$. Payoffs excluding endowments are presented as (payoff A, payoff B, payoff C). To facilitate understanding and see how similar the different conditions are, the figure presents final payoffs. In the experiment, subjects were not presented with final payoffs. Regardless of what A chose, in the second stage subjects were presented with identical choices and payoff consequences as the No Transfer Condition. Coordinated outcomes are shaded.

A strategy for $\mathrm{A}$ in $\Gamma_{V}$ is, for example, $\{$ Transfer, Low, High $\}$ or $(T L H)^{5}$, meaning A transfers to C, followed by Low if she did not transfer and High if she did. A strategy for B is, for example, $\{\underline{H i g h}, \underline{L o w}\}$ or $(\underline{H L})$, meaning B plays $\underline{H i g h}$ if A did not transfer and Low if she did.

To identify how a voluntary transfer option affects coordination, we compare second stage coordination rates in the Voluntary Transfer Game with those in the No Transfer Game. To understand why transfers affect coordination we compare behaviour in the Voluntary Transfer Game to the Mandatory Transfer Game (which we now define).

In the Mandatory Transfer Game, $\Gamma_{M}$, a transfer is made from A to $\mathrm{C}$, then $\mathrm{A}$ and $\mathrm{B}$ play the same battle of the sexes as in the second stage of the No Transfer Game. Payoffs are given by the outcome of the coordination game adjusted for the transfer. In terms of final payoffs, the second stage of this game is identical to the second stage of the Voluntary Transfer Game conditional on A having chosen to transfer. As in the previous condition, only stage payoffs were presented in the experiment, thus the possible choices and payoff consequences in the second stage were identical to those in the other two conditions.

\footnotetext{
${ }^{5}$ We may refer to actions, strategies, strategy profiles and histories by acronyms specifying the first letter of each component action: T=Transfer; $N=$ No Transfer; H=High; L=Low; $\underline{H}=\underline{H i g h} ; \underline{L}=\underline{\text { Low }}$.
} 
Figure 4: The Mandatory Transfer Condition

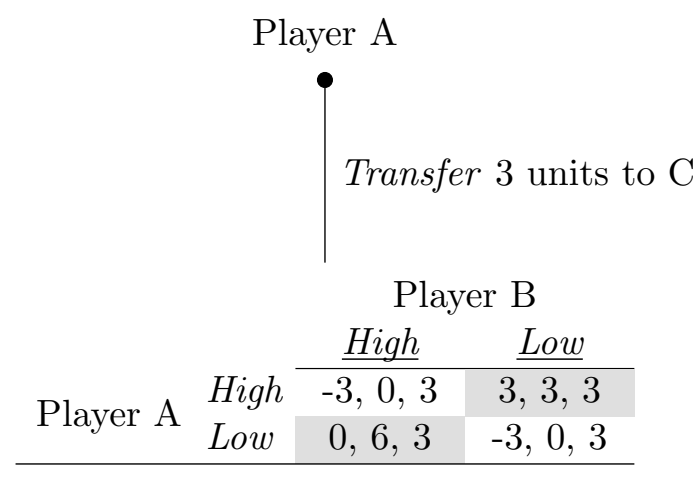

Notes: This figure describes the game form played in the Mandatory Transfer Condition, the Mandatory Transfer Game, $\Gamma_{M}$. Payoffs excluding endowments are presented as (payoff A, payoff B, payoff C). The figure presents final payoffs. In the experiment, subjects were not presented with final payoffs, but stage payoffs. That is the second stage, possible choices and payoff consequences were identical to the No Transfer Condition. Coordinated outcomes are shaded.

As we will explain in the following subsection, some theories (distributive preferences) predicting that a transfer will improve coordination apply regardless of whether the transfer is voluntary or mandatory, whereas others (forward induction and signalling) only apply if the transfer is voluntary. Thus by comparing coordination rates in the Mandatory Transfer Condition to those in the Voluntary Transfer Condition we can identify which of the two types of theory explain why redistribution improves coordination.

Our design choice to decouple second stage and final payoffs is important in ensuring that the second stage coordination game is identical across conditions. Subjects in all conditions are presented with identical second stage choices and second stage payoff possibilities. For example, in the Mandatory Transfer Condition, subjects are asked for a choice given that the payoff implications are the stage payoffs (i.e. Figure 1 payoffs), not final payoffs (i.e. Figure 4 payoffs). This decoupling of stage payoffs is important as it limits the extent to which gravitate towards the perfectly equal outcome. If it were not for this, we may overestimate the coordinating power of redistribution in our experiment due to the salience of the perfectly equal payoff possibility (Schelling 1960; Van Huyck et al. 1992; López-Pérez et al. 2015; Bett et al. 2016).

In all conditions, subjects interact anonymously via computers, are randomly alternated between each of the three player roles, and interact repeatedly with randomly matched groups. This design removes dynamic incentives (e.g. reputation-building), but allows for learning to occur.

We now analyse the game in each condition to form predictions. 


\subsection{Theoretical predictions}

Where possible, we identify conditions under which players coordinate in each game.

\section{The No Transfer Game}

$\Gamma_{N}$ has two pure strategy Nash Equilibria (NE): High-Low and Low-High. The symmetry of the game makes coordination particularly difficult. The mixed strategy equilibrium involves each player choosing high with probability $\frac{2}{3} .{ }^{6}$ While previous research suggests that player/action labels (Crawford et al. 2008) or that A is forced to not transfer before the coordination game (cf. Huck and Müller 2005) may improve coordination, the effects are likely to be relatively small. We thus predict the mixed strategy NE will be played.

Observation 1 (Mixed strategy $\mathbf{N E}$ of $\Gamma_{N}$ ). In the mixed strategy $N E$ of $\Gamma_{N}$, A plays

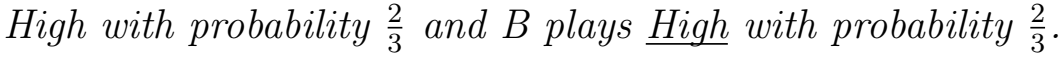

\section{The Voluntary Transfer Game}

First notice that $\Gamma_{V}$ has multiple equilibria (there are five pure strategy SPE: $\{N H H, \underline{L L}\}$, $\{N H L, \underline{L H}\},\{T L H, \underline{H L}\},\{N L H, \underline{H L}\}$ and $\{N L L, \underline{H H}\})$. Despite this, there are at least three reasons to believe coordination is possible.

\section{Distributive preferences}

There is considerable experimental evidence that individuals have distributive preferences (e.g. Fehr and Schmidt 2006). Such preferences cannot lead to coordination in the No Transfer Game given how symmetric the game is, but they may do so in the Voluntary Transfer Game. To illustrate, we identify the effect of one important motivation: inequity-aversion, where players have a preference for minimising payoff differences (Fehr and Schmidt 1999).

Let $I$ be the set of players, $\pi_{i}$ be $i$ 's payoff and $\pi$ be a payoff vector. Suppose player $i$ 's preferences are represented by

$$
U_{i}(\pi)=\pi_{i}-\frac{\alpha_{i}}{2} \sum_{j \in I \backslash\{i\}} \max \left\{\pi_{j}-\pi_{i}, 0\right\}-\frac{\beta_{i}}{2} \sum_{j \in I \backslash\{i\}} \max \left\{\pi_{i}-\pi_{j}, 0\right\},
$$

where $\alpha_{i}$ and $\beta_{i}$ represent $i$ 's sensitivity to disadvantageous and advantageous inequality respectively, satisfying $0 \leq \beta_{i} \leq \alpha_{i}{ }^{7}$

\footnotetext{
${ }^{6}$ Each player must be indifferent between their action choices in a non-degenerate mixed strategy

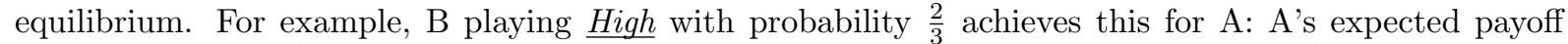
from High is then $\frac{2}{3}(0)+\frac{1}{3}(6)=2$ which equals her expected payoff from Low, $\frac{2}{3}(3)+\frac{1}{3}(0)=2$.

${ }^{7}$ We allow for stronger preferences against inequity than Fehr and Schmidt (1999) in that we do not impose $\beta_{i}<1$.
} 
Observation 2 (Inequity-aversion in $\Gamma_{V}$ ). For all $\alpha_{A}+\beta_{A} \geq 2$ and $\beta_{B}>2+\frac{\alpha_{A}}{2}$,

a. if $\beta_{A}<\frac{4}{3}$, there exists a unique SPE, $\{T H H, \underline{H L}\}$,

b. if $\beta_{A} \geq \frac{4}{3}$, the set of SPE is $(\{T H H, \underline{H L}\},\{T L H, \underline{L L}\})$.

In either case, THL is played on path in all SPE.

Proof: See Appendix A.

The result implies that coordination is possible in the Voluntary Transfer Game, if players are sufficiently inequity-averse. To understand why Transfer then High-Low is played, reason as follows. First note that if B is sufficiently inequity-averse then the unique NE in the subgame following A choosing to Transfer is High-Low. To see why Low-High is not a NE in this subgame, consider B's incentives. If B were to deviate to Low, his material payoff would fall, thus his utility would decrease. However, the more equitable payoff distribution implies that his utility would increase. If $\mathrm{B}$ cares sufficiently about avoiding inequities, the latter is larger than the former and thus B would deviate, implying Low- $\underline{\text { High }}$ is not a NE in this subgame.

Second, if both players are sufficiently inequity-averse, in the subgame following $N_{o}$ Transfer only High- $\underline{\text { High }}$ and Low-Low can be NE as they give perfect payoff equality (all players receive a payoff of zero). Given optimal second stage behaviour, in the first stage A would choose to Transfer as it gives a higher material payoff and has no effect on payoff differentials.

\section{Forward induction}

Our second route to coordination relies on the sequential nature of the Voluntary Transfer Game. In the No Transfer Game, B does not learn anything from A's first-stage choice, since A was forced to not transfer (i.e. had no choice). In the Voluntary Transfer Game, A actively chooses whether or not to transfer. Forward induction is the idea that one can use a player's past choices to predict her future choices (van Damme 1989; BenPorath and Dekel 1992). A's choice to transfer or not may signal A's intended behaviour in the battle of the sexes to B. The availability of such signals may help coordination.

Our next observation is based on such forward induction reasoning.

Observation 3 (Forward induction in $\Gamma_{V}$ ). The forward induction solutions of $\Gamma_{V}$ are $\{N H H, \underline{L L}\}$ and $\{N H L, \underline{L L}\}$. Forward induction implies a unique path of play, NHl.

Proof: See Appendix A.

The intuition behind why forward induction predicts No transfer followed by High$\underline{L o w}$ is identical to that found in money burning games (e.g. Ben-Porath and Dekel 1992). To see it here, consider the following two steps of reasoning: 
Step 1: It cannot be rational for A to play Transfer followed by Low, as her payoff would be weakly greater if she plays No transfer followed by Low. Given this, if B observes A playing Transfer he must infer that A plans to then play High, thus B best-responds with $\underline{\text { Low }}$ and A gets a payoff of three.

Step 2: Given that A can get a payoff of three for sure by playing Transfer, it cannot be that A plays No Transfer and then plans to play Low, as she would then get weakly less than three. Thus by choosing No Transfer A signals to B that she intends to then play High, to which B best-responds with $\underline{\text { Low}}$, securing A a payoff of six.

\section{Signalling altruism}

Our final path to coordination involves a different form of signalling. The model below (based on Levine 1998, cf. Benabou and Tirole 2006) captures how A may use her transfer choice to signal her privately known degree of altruism to B. Doing so may help coordination.

Let A's preferences be represented by the utility function $U_{A}=\pi_{A}+\alpha_{\tau}\left(\pi_{B}+\pi_{C}\right)$, where $\tau \in\{g, m\}$ is A's altruism type (either generous, $g$, or miserly, $m$ ) and $\alpha_{g}>\alpha_{m}=0$. Let $p \in\{0,1\}$ be the prior probability that A is of type $g$. A strategy for A is denoted by $s_{i}(\tau)$, a pure strategy for each type of A. Let B maximise his material payoff. Given these preferences, we identify sequentially strict separating Perfect Bayesian Equilibria (PBE).

Observation 4 (Signalling altruism in $\Gamma_{V}$ ). For $\alpha_{g}>1$, there exists a unique sequentially strict separating PBE described as follows: $s_{A}^{*}(g)=T H L, s_{A}^{*}(m)=N H L$ and $s_{B}^{*}=\underline{L H}$.

Proof: See Appendix A.

The result predicts that a generous type A would choose Transfer and then Low- $\underline{\text { High }}$ will be played; and a miserly type A would choose No Transfer and High-Low would be played.

For some intuition, reason as follows. First note that neither type of A would want to mis-coordinate (play High-High or Low-Low) as unilateral deviation can increase both own and others' material payoffs. Comparing payoffs in the two remaining coordination game outcomes, notice that $\mathrm{A}$ and $\mathrm{B}$ are playing a zero sum game. Note also that a generous A is sufficiently altruistic such that she would be willing to sacrifice a unit of her own payoff to increase the other player's payoff by a unit. This means transferring and coordinating on Low-High is a credible signal of her type as a miserly type would not be willing to make such a sacrifice. Conversely, this makes not transferring then coordinating on High-Low a credible signal for the miserly type. 
Table 1 summarises our predictions for the Voluntary Transfer Game.

Table 1: Summary of predictions in $\Gamma_{V}$

\begin{tabular}{|c|c|c|}
\hline & Path of play & Off-path behaviour \\
\hline Inequity-aversion & Transfer-High-Low & High- $\underline{\text { High }}$ or Low-Low \\
\hline Forward induction & No Transfer-High-Low & High-Low \\
\hline $\begin{array}{l}\text { Signalling altruism A altruistic } \\
\text { if A not altruistic }\end{array}$ & $\begin{array}{l}\text { Transfer-Low- } \underline{\text { High }} \\
\text { No Transfer-High-Low }\end{array}$ & $\begin{array}{l}\text { High-Low } \\
\text { Low- } \underline{\text { High }}\end{array}$ \\
\hline
\end{tabular}

Notes: The table summarises the path of play and off-path behaviour predicted by each theory.

\section{The Mandatory Transfer Game}

Notice that the coordination problem still exists in $\Gamma_{M}$ (High-Low and Low- $\underline{\text { High }}$ are both still NE), coordination may nonetheless be possible as we explain below.

Neither forward induction nor signalling imply coordination in the Mandatory Transfer Game as A does not make an active choice before the coordination game. Inequity-aversion can however imply coordination. This allows us to separate the effect of inequity-aversion from forward induction and signalling. Recall the definitions for inequity-aversion (Fehr and Schmidt 1999) presented in our analysis of the Voluntary Transfer Game.

Observation 5 (Inequity-aversion in $\Gamma_{M}$ ). For all $\beta_{B}>2+\frac{\alpha_{B}}{2}$, High-Low is the unique $N E$ of $\Gamma_{M}$.

Proof: See Appendix A.

The result implies that coordination is possible in the Mandatory Transfer Game if B is sufficiently inequity-averse. The intuition behind this result is identical to that explaining why inequity-aversion can imply coordination in the subgame following Transfer in the Voluntary Transfer Game (see the first paragraph after Observation 2).

\subsection{Hypotheses}

Using the predictions from 2.1, we now formulate hypotheses about whether voluntary redistribution can improve coordination, and if so, why. Since none of our lines of reasoning predicted players playing High-High or Low-Low on path, we refer to coordination as playing either High-Low or Low- $\underline{\text { High }}$. 


\section{Does voluntary redistribution improve coordination?}

Given that none of our theoretical motivations suggested coordination in the No Transfer Condition, while several did in the Voluntary Transfer Condition (Observations 2-4 and Table 1), we have the following hypothesis.

Hypothesis 1. Coordination rates will be higher in the Voluntary Transfer Condition than the No Transfer Condition.

One might think that coordination is very likely when there are several reasons why players coordinate in the Voluntary Transfer Condition. However, this is not necessarily the case. The problem is that the behaviour to coordinate on is dependent on the explanation for coordination. For example, how should players coordinate if A plays Transfer? If players were inequity-averse, they should coordinate on High-Low (Observation 2). By contrast if A was signalling her altruism, they should coordinate on Low-High (Observation 4).

At the start of the experiment, subjects do not know others' preferences and reasoning processes, so coordination rates will presumably be relatively low (perhaps 50\%) due to the confusions just highlighted. Importantly, subjects interact repeatedly in our experiment, albeit with stranger matching. Through repetition they would learn about others' preferences and reasoning. Norms of how they coordinate after A's choice to Transfer or Not Transfer will develop, implying coordination rates will increase over time in the Voluntary Transfer Condition. Thus the assumptions behind our predictions are more likely to hold towards the end of the experiment.

\section{Why might voluntary redistribution improve coordination?}

The remaining hypotheses are based on instances where different theoretical motivations make different predictions. This allows us to discriminate between different explanations as to why voluntary redistribution might improve coordination.

Recall that inequity-aversion predicted coordination in both the mandatory and the voluntary conditions, while forward induction and signalling predicted coordination only in the voluntary condition. This motivates our second hypothesis.

Hypothesis 2. Coordination rates in the Voluntary Transfer Condition will be:

a. (Inequity-aversion) No different from those in the Mandatory Transfer Condition; b. (Forward induction and signalling) Higher than those in the Mandatory Transfer Condition.

Comparing coordination in the No Transfer Condition with the Voluntary Transfer Condition conditional on A having chosen not to transfer, we identify the coordination effect of a voluntary choice to not transfer. Again our different theories make different predictions. 
Hypothesis 3. Coordination rates in the Voluntary Transfer Condition conditional on A choosing not to transfer will be:

a. (Inequity-aversion) No different from those in the No Transfer Condition;

b. (Forward induction and signalling) Higher than those in the No Transfer Condition.

A similar hypothesis can be stated for the comparison between the Mandatory Transfer Condition and the Voluntary Transfer Condition conditional on a transfer.

Hypothesis 4. Coordination rates in the Voluntary Transfer Condition conditional on A choosing to transfer will be:

a. (Inequity-aversion) No different from those in the Mandatory Condition;

b. (Forward induction and signalling) Higher than those in the Mandatory Condition.

Finally, each theory predicts different choices within the Voluntary Transfer Condition, giving our last hypothesis.

Hypothesis 5. In the Voluntary Transfer Condition subjects play:

a. (Inequity-aversion) Transfer-High-Low and either High-High or Low-Low off-path;

b. (Forward induction) No Transfer-High-Low and Low-High off-path;

c. (Signalling altruism) If $A$ is altruistic, then Transfer-Low-High and High-Low off-

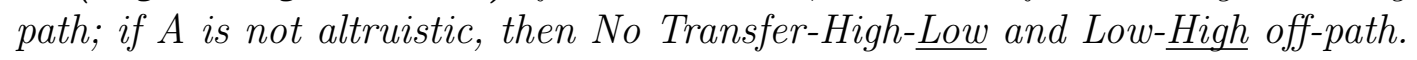

As already argued, learning could be very important in the Voluntary Transfer Condition. The hypotheses are thus most likely to hold later in the experiment.

\subsection{Experimental procedures}

The experiment was run using z-tree (Fischbacher 2007) at the MELESSA laboratory of the Ludwig Maximilian University of Munich, Germany. We conducted six sessions, two per condition. A session lasted approximately 60 minutes and involved 24 subjects (144 in total). Subjects were recruited via ORSEE (Greiner 2015) and participated in only one session.

Subjects were randomly allocated to a computer station with printed instructions (see Appendix C). The instructions described a three player game with a Player A who could make a choice before playing a battle of the sexes game with Player B. The instructions highlighted the existence of a passive third player, C. These basic descriptions of the game were identical for all subjects and conditions. Condition and decision differences were only specified on-screen.

In each session we ran one condition only. In the No Transfer and Mandatory Transfer Conditions, subjects were told on-screen that the first stage choice was not made available 
to A and that Transfer or No Transfer had been imposed. Subjects in Voluntary Transfer Condition were made aware that Transfer or No Transfer in the first stage would be actively chosen by A.

In each session, the condition game was repeated for 10 periods, where each period consisted of three subperiods. Each subperiod ran as follows: a group of three subjects was randomly formed, each subject was assigned a player role and the condition game was played once; then groups were broken up. Each subject played all three player roles within a period: a different one in each subperiod, in a randomly determined order and with different randomly chosen partners. This ensured that subjects played each role equally often and stranger matching across subperiods. Re-matching subjects at each subperiod allows us to observe whether subjects systematically modify their behaviour without being credibly able to influence others' future choices by acting strategically. Subjects were only informed of the outcomes of the three subperiods at the end of each period.

Earnings were measured in Tokens throughout the experiment. For each subject, one subperiod was randomly selected and the subject earned whatever their final payoff was in that subperiod, we thus avoid income effects. Together with the stranger matching protocol, this yields 480 independent observations per condition using each coordinating couple A and B in each period as an observation unit. ${ }^{8}$

The experiment began after the instructions were read out loud and questions answered privately. After the experiment we elicited social value orientations and collected data on the subject's gender, age and general trust attitude on a Likert scale. Average earnings were approximately $€ 14$.

\section{Results}

In this section we present analyses of the hypotheses presented in Section 2.2. Our main focus is on how a voluntary transfer affects coordination and the explanation for the effect. $^{9}$

\footnotetext{
${ }^{8}$ Assuming a $10 \%$ difference centred around 0.5 in the proportion of times subjects coordinate on a specific outcome across conditions gives a power of 0.87 at $5 \%$ significance level. Using the frequencies with which subjects in Huck and Müller (2005) coordinate on PY (High-Low in our paper) across the battle of the sexes and Burn conditions in their study, our power is close to 1.

${ }^{9}$ Only experimental data and analysis relevant to testing our hypotheses are presented in the main text. Further data analysis is found in Appendix B.
} 


\subsection{Does voluntary redistribution improve coordination?}

For an initial impression of how voluntary redistribution affects coordination, Figure 5 shows coordination rates by condition and Figure 6 the rates over time. ${ }^{10}$

Figure 5: Coordination rates by condition

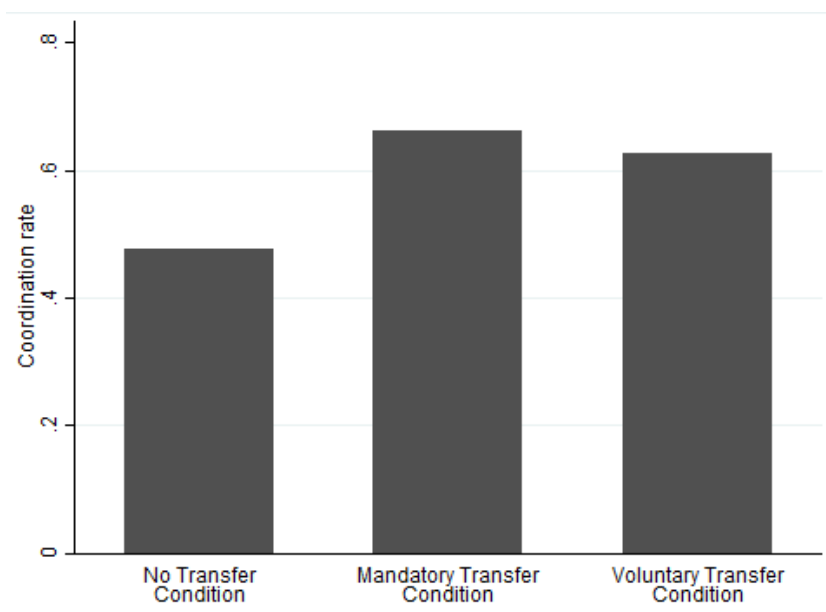

Notes: This figure depicts the coordination rate by condition. The coordination rate is the share of times that High- $\underline{\text { Low }}$ or Low- $\underline{\text { High }}$ was played.

Figure 6: Coordination rates by condition over time

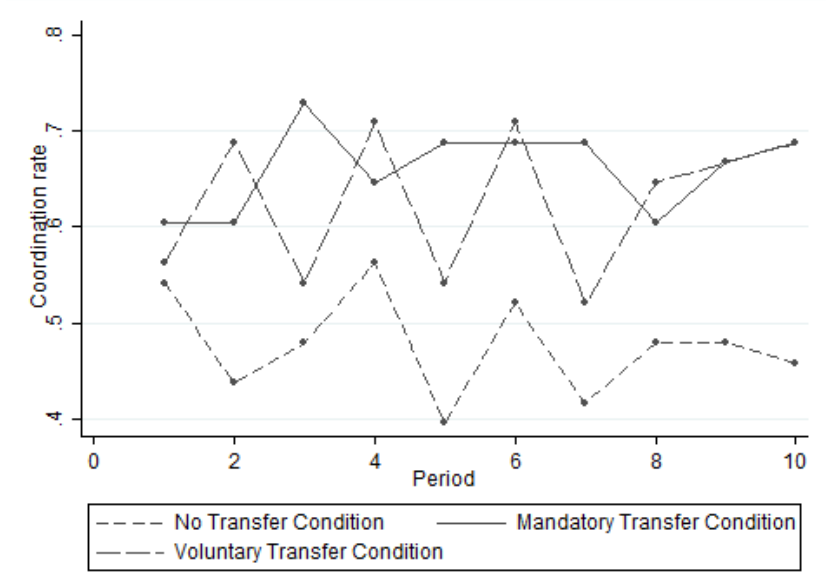

Notes: This figure depicts the coordination rate by condition and period. The coordination rate is the share of times that High-Low or Low- $\underline{\text { High }}$ was played.

\footnotetext{
${ }^{10}$ Randomisation into conditions was made at session level as discussed in Section 2.1. We thus test for differences across conditions in subject observables. Subjects' genders, ages, occupations and SVO-scores are not significantly different across conditions (Kruskall-Wallis tests do not reject the null of equality for gender $(p=0.341)$, age $(p=0.483)$, occupation $(p=0.921)$ and SVO score $(p=0.428))$.
} 


\section{Testing Hypothesis 1}

The coordination rate in the No Transfer Condition was $47.7 \%$, whereas that in the Voluntary Transfer Condition was $62.7 \%$, a statistically significant difference (Fisher's Exact test, $p<0.001)$.

As discussed in Section 2.2 it was not obvious that coordination would be higher early on in the Voluntary Transfer Condition due to the different paths to coordination predicted theoretically (Table 1). However Figure 6 suggests that the difference between the Voluntary Transfer and No Transfer Conditions emerges within a few periods. This is particularly striking given that we used a stranger matching design. Whether this difference is statistically significant in each period is hard to see from the figure.

To better see when the Voluntary Transfer Condition gives a statistically significant higher coordination rate, we pooled data from the No Transfer and Voluntary Transfer Conditions and estimated period-wise Linear Probability Models where the dependent variable was a dummy for coordination. The independent variables were a dummy for the Voluntary Transfer Condition and controls for subperiods. We used robust standard errors clustered at session level. Figure 7 presents the coefficient estimates and 95\% confidence intervals from the period-wise Linear Probability Models.

Figure 7: The effect of a voluntary transfer option on coordination

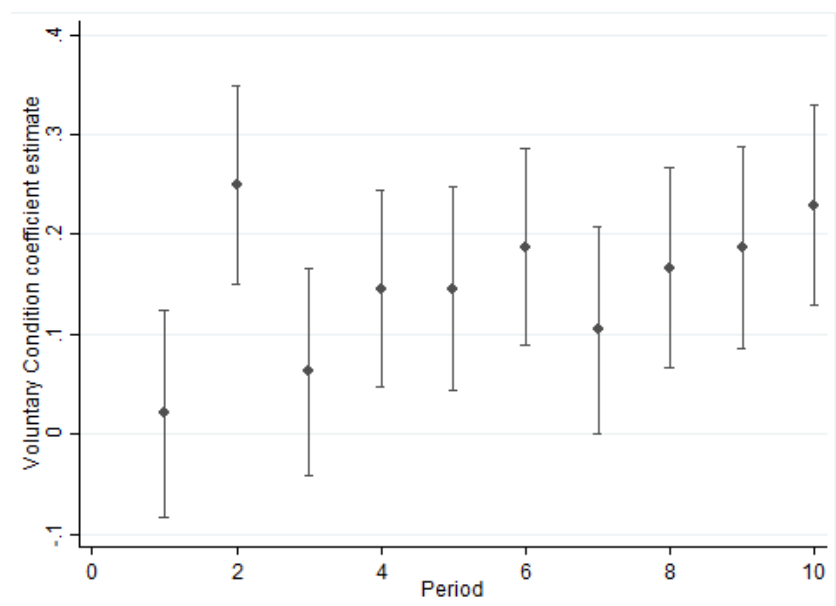

Notes: This figure displays treatment effect estimates and 95\% confidence intervals from periodwise OLS regressions where the dependent variable is Coordination (a dummy for whether or not the group coordinated). Data from the No Transfer and Voluntary Transfer Conditions were pooled, the treatment is a dummy variable for the Voluntary Transfer Condition. Controls for the subperiod were included and robust standard errors clustered at session level were used.

Notice the slight upward trend over time in the difference between coordination in the No Transfer and Voluntary Transfer Conditions and that the difference becomes sta- 
tistically significant by period four. ${ }^{11}$ The difference is driven primarily by coordination increasing in the Voluntary Transfer Condition and that in the No Transfer Condition remaining roughly constant (see Figure 6). This is consistent with our conjecture that learning via repeated interaction would be particularly helpful for coordination in the Voluntary Transfer Condition.

All of the above provide strong support for Hypothesis 1.

Result 1. Coordination rates in the Voluntary Transfer Condition are higher than in the No Transfer Condition. The difference increases over time.

The result is clear evidence that a voluntary redistribution option can improve coordination. Next we investigate why a voluntary redistribution option improves coordination.

\subsection{Why does voluntary redistribution improve coordination?}

As explained in Section 2, different theories predict different behaviour in our conditions. We are thus able to discriminate between competing explanations for why a voluntary transfer option increases coordination. To separate inequity-aversion from forward induction and signalling we examine behaviour across all three conditions (see Hypotheses 2-4). To separate forward induction from signalling we examine behaviour within the Voluntary Transfer Condition (see Hypothesis 5).

\section{Testing Hypothesis 2}

Whether a transfer is voluntary or mandatory has no effect on the possible payoff distributions, thus inequity-aversion predicted no difference in coordination between the Voluntary and Mandatory Transfer Condition. In sharp contrast, for forward induction or signalling to increase coordination the transfer must be voluntary, thus these theories predicted higher coordination in the Voluntary condition than the Mandatory condition.

We find that the difference in coordination rates between the Mandatory and Voluntary Transfer Conditions is not statistically significant (Fisher's Exact test, $p<0.001$ ), implying that inequity-aversion explains why transfers increase coordination.

Despite there being no difference on aggregate, Figure 6 suggests dynamic differences, driven primarily by the very volatile coordination rate in the Voluntary Transfer Condition in the first two-thirds of the experiment. To better see when the Voluntary Transfer Condition gives a statistically significant higher coordination rate, we pooled data from the Mandatory and Voluntary Transfer Conditions and estimated period-wise Linear Probability Models where the dependent variable was a dummy for coordination. The independent variables were a dummy for the Voluntary Transfer Condition and controls for subperiods. We used robust standard errors clustered at session level. Figure

\footnotetext{
11 We obtain qualitatively similar results when we: estimate Logit or Probit regressions; do or do not include robust standard errors clustered at session level and subperiod controls; estimate subperiodperiod-wise rather than period-wise models; bootstrap standard errors.
} 
8 presents the coefficient estimates and 95\% confidence intervals from the period-wise Linear Probability Models.

Figure 8: The effect of a voluntary- relative to a mandatory transfer option on coordination

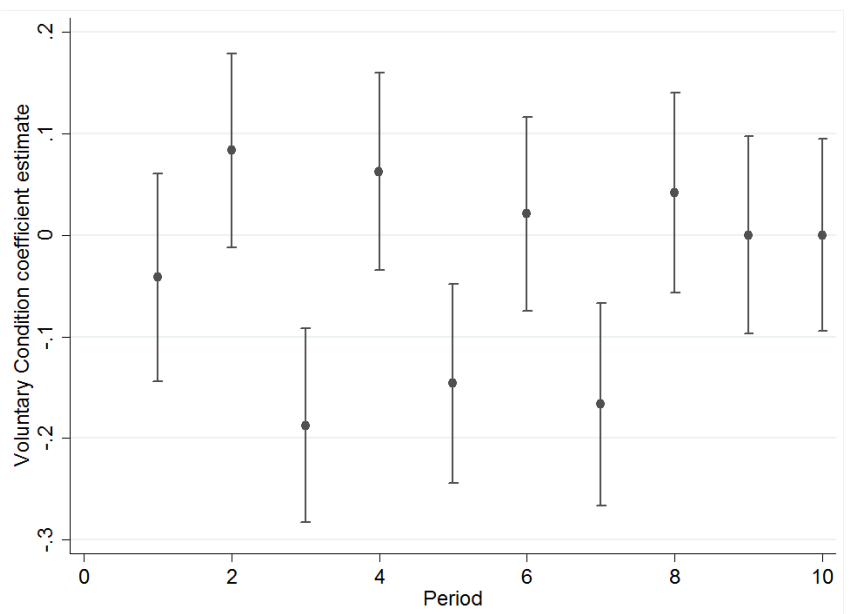

Notes: This figure displays treatment effect estimates and 95\% confidence intervals from periodwise OLS regressions where the dependent variable is Coordination (a dummy for whether or not the group coordinated). Data from the Mandatory Transfer and Voluntary Transfer Conditions were pooled, the treatment is a dummy variable for the Voluntary Transfer Condition. Controls for the subperiod were included and robust standard errors clustered at session level were used.

Differences in coordination rates are not statistically significant between the conditions in most periods. ${ }^{12}$ It is hard to see any dynamic patterns at this level of aggregation.

To further investigate whether there are any dynamic patterns, we again pooled data from the Mandatory and Voluntary Transfer Conditions, but this time estimated Linear Probability Models period-subperiod-wise, rather than period-wise. Once again, the dependent variable was a dummy for coordination; the independent variable was a dummy for the Voluntary Transfer Condition and we used robust standard errors clustered at session level. Figure 9 presents coefficient estimates and confidence intervals from this analysis.

\footnotetext{
${ }^{12}$ We obtain qualitatively similar results when we: estimate Logit or Probit regressions; do or do not include robust standard errors clustered at session level and subperiod controls; bootstrap standard errors.
} 
Figure 9: The effect of a voluntary- relative to a mandatory transfer option on coordination

(by period-subperiod)

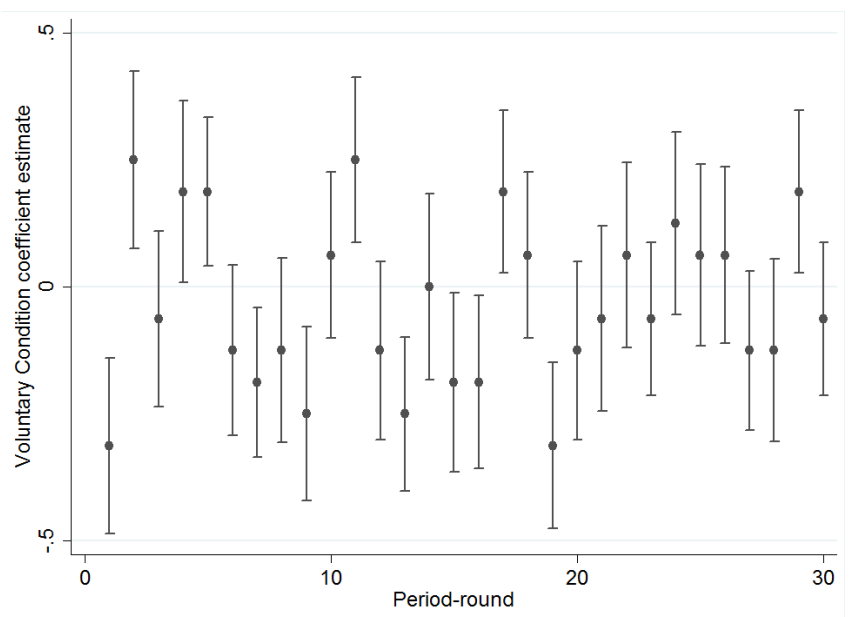

Notes: This figure displays treatment effect estimates and 95\% confidence intervals from periodsubperiod-wise OLS regressions where the dependent variable is Coordination (a dummy for whether or not the group coordinated). Data from the Mandatory Transfer and Voluntary Transfer Conditions were pooled, the treatment is a dummy variable for the Voluntary Transfer Condition. Robust standard errors clustered at session level were used.

The difference in coordination rates is clearly a lot less in later period-subperiods than earlier period-subperiods. For example, in the first two thirds of the experiment (periodsubperiods 1-20), there is a statistically significant difference in coordination between the two conditions in 12 out of 20 period-subperiods. In the final third (period-subperiods 21-30) there was only one such period-subperiod. ${ }^{13}$ This is consistent with the idea that after learning the two transfer conditions have the same coordination rate, in line with inequity-aversion, but not forward induction and signalling.

Result 2. Coordination rates in the Voluntary Transfer Condition are not significantly different from the Mandatory Transfer Condition. This is particularly true in later periods.

We cannot yet conclude that forward induction and signalling do not play a role in explaining why voluntary redistribution improves coordination. To more clearly identify their role, if any, we test Hypotheses 3-5.

\footnotetext{
${ }^{13}$ We obtain qualitatively similar results when we: estimate Logit or Probit regressions; do or do not include robust standard errors clustered at session level; bootstrap standard errors.
} 


\section{Testing Hypothesis 3}

Whether A chooses not to transfer or has no option to transfer does not affect the possible payoff distributions. Thus inequity-aversion predicted identical coordination in the No Transfer Condition and the Voluntary Transfer Condition conditional on A choosing to not transfer. By contrast, both forward induction and signalling rely on an active choice to not transfer, thus they predicted higher coordination in the Voluntary Transfer Condition conditional on A choosing to not transfer than the No Transfer Condition.

We find that the coordination rate in the Voluntary Transfer Condition conditional on A choosing not to transfer is $42.7 \%$. This is not significantly different from the $47.7 \%$ coordination in the No Transfer Condition (Fisher's Exact test, $p=0.389$ ), consistent with inequity-aversion.

To identify any dynamic patterns, we pooled data from the No Transfer Condition with that from the and Voluntary Transfer Condition conditional on A not transferring and estimated period-wise Linear Probability Models where the dependent variable was a dummy for coordination. The independent variables were a dummy for the Voluntary Transfer Condition and controls for subperiods. We used robust standard errors clustered at session level. Figure 10 presents the coefficient estimates and 95\% confidence intervals from the period-wise Linear Probability Models. Note that in most periods there is not a statistically significant difference in coordination across the two conditions.

Figure 10: The effect of a voluntary no transfer relative to a forced no transfer on coordination

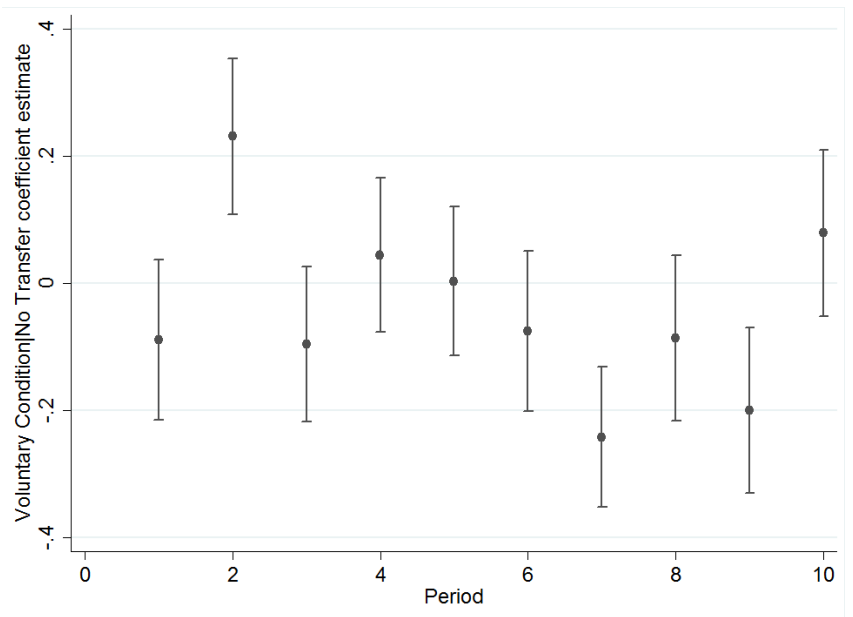

Notes: This figure displays treatment effect estimates and $95 \%$ confidence intervals from periodwise OLS regressions where the dependent variable is Coordination (a dummy for whether or not the group coordinated). Data from the No Transfer Condition and Voluntary Transfer Condition given No Transfer was chosen were pooled, the treatment is a dummy variable for the Voluntary Transfer Condition given Transfer was chosen. Controls for the subperiod were included and robust standard errors clustered at session level were used. ${ }^{11}$ 
Result 3. Coordination rates in the Voluntary Transfer Condition conditional on $A$ choosing not to transfer are not significantly different from those of the No Transfer Condition.

This is our second result consistent with inequity-aversion and inconsistent with forward induction and signalling. So far there is no evidence that forward induction or signalling explain why a voluntary redistribution option increases coordination.

\section{Testing Hypothesis 4}

Whether A chooses to transfer or is coerced to transfer does not affect the possible payoff distributions. Thus inequity-aversion predicted identical coordination in the Mandatory Transfer Condition and the Voluntary Transfer Condition conditional on A choosing to transfer. By contrast, both forward induction and signalling rely on an active choice to transfer, thus they predicted higher coordination in the Voluntary Transfer Condition conditional on A choosing to transfer than the Mandatory Transfer Condition.

We find that the coordination rate in the Voluntary Transfer Condition conditional on A choosing to transfer is $82.0 \%$. This is significantly higher than the $66.4 \%$ coordination in the Mandatory Transfer Condition (Wilcoxon Rank Sum test, $p<0.001$ ). Unlike previous pieces of evidence, this is consistent with forward induction and signalling, but inconsistent with inequity-aversion.

To identify any dynamic differences, we pooled data from the Mandatory Transfer Condition with that from the and Voluntary Transfer Condition conditional on A transferring and estimated period-wise Linear Probability Models where the dependent variable was a dummy for coordination. The independent variables were a dummy for the Voluntary Transfer Condition and controls for subperiods. We used robust standard errors clustered at session level. Figure 11 presents the coefficient estimates and 95\% confidence intervals from the period-wise Linear Probability Models. Notice that the difference in coordination between the two conditions is increasing over time. ${ }^{11}$ 
Figure 11: The effect of a voluntary transfer relative to a forced transfer on coordination

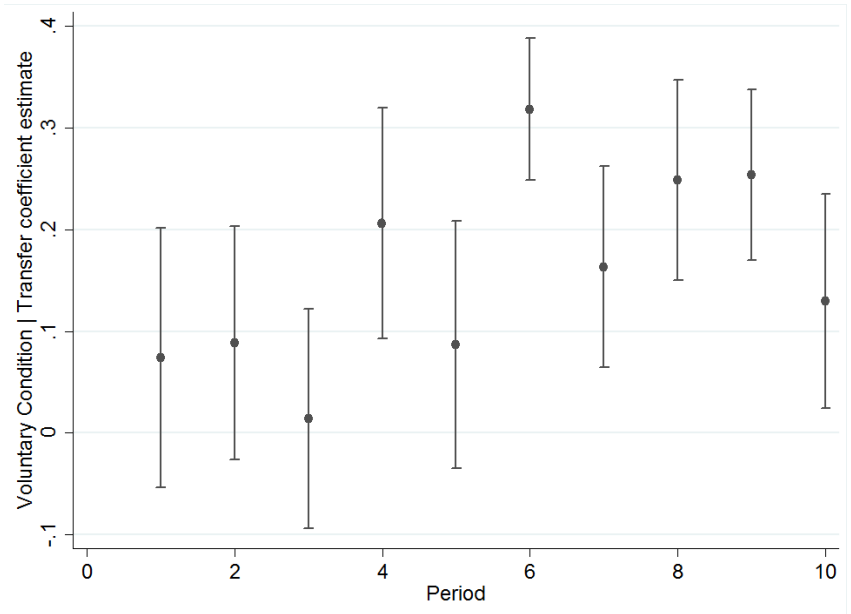

Notes: This figure displays treatment effect estimates and $95 \%$ confidence intervals from periodwise OLS regressions where the dependent variable is Coordination (a dummy for whether or not the group coordinated). Data from the Mandatory Transfer Condition and Voluntary Transfer Condition given Transfer was chosen were pooled, the treatment is a dummy variable for the Voluntary Transfer Condition given Transfer was chosen. Controls for the subperiod were included and robust standard errors clustered at session level were used.

Result 4. Coordination rates in the Voluntary Transfer Condition conditional on A choosing to transfer are significantly higher than those in the Mandatory Transfer Condition. This difference increases over time.

As explained earlier, inequity-aversion predicted that a transfer should have the same effect whether it is voluntary or coerced. Result 4 is clearly inconsistent with this prediction. It is however consistent with forward induction and signalling, which both predicted a voluntary transfer can coordinate behaviour better than a coerced one.

This is our first clear evidence suggesting that forward induction or signalling may explain why a voluntary redistribution option increases coordination. To further discriminate between these explanations, we now test our hypothesis on behaviour within the Voluntary Transfer Condition.

\section{Testing Hypothesis 5}

Recall that different theories made different predictions for on- and off-path behaviour in the Voluntary Transfer Condition (see Table 1 and Hypothesis 5). Examining choices in this condition can thus help us identify which theory best explains why a voluntary transfer option increases coordination. Table 2 presents the relative frequencies of choices in the Voluntary Transfer Condition. 
Table 2: Relative frequency of choices and outcomes in the Voluntary Transfer Condition

\begin{tabular}{|c|c|c|c|c|}
\hline \multicolumn{5}{|c|}{ Panel A: Aggregate } \\
\hline 480 obs. & & High & $\begin{array}{c}\text { Player } \\
\text { Low }\end{array}$ & \\
\hline \multirow{2}{*}{ Player A } & High & 31.46 & 55.62 & 87.08 \\
\hline & Low & 7.08 & 5.83 & 12.91 \\
\hline \multicolumn{5}{|c|}{$38.54 \quad 61.45$} \\
\hline \multicolumn{5}{|c|}{ Panel B: Following A choosing not to transfer } \\
\hline 246 obs. & & & Player & \\
\hline \multirow{4}{*}{ Player A } & & High & Low & \\
\hline & High & 45.12 & 32.11 & 77.23 \\
\hline & Low & 10.57 & 12.19 & 22.76 \\
\hline & & 55.69 & 44.3 & \\
\hline \multicolumn{5}{|c|}{ Panel C: Following A choosing to transfer } \\
\hline 234 obs. & & High & $\begin{array}{c}\text { Player } \\
\text { Low }\end{array}$ & \\
\hline \multirow{2}{*}{ Player A } & High & $\overline{17.09}$ & $\overline{80.34}$ & 97.43 \\
\hline & Low & 1.71 & 0.85 & 2.56 \\
\hline & & 18.8 & 81.19 & \\
\hline
\end{tabular}

Notes: This table displays the percentage of times each second-stage outcome was played in the Voluntary Transfer Condition: on aggregate (Panel A), following A choosing not to transfer (Panel B) and following A choosing to transfer (Panel C). The percentage of times a particular action was played is stated on the right and below each table. Coordinated outcomes are shaded.

Notice that A chose to transfer 48.8\% (234/480) of the time. In Section 2.1 we focused on identifying conditions implying a unique equilibrium. Aside from our model of signalling altruism where due to incomplete information both of A's first stage action choices are on path in the unique equilibrium, all other models gave degenerate paths of play. At this aggregate level, it is thus impossible that a single model can explain all subject behaviour. We examine second stage behaviour in detail to see which model best fits the data.

First consider behaviour following A choosing to not transfer. As already noted, coordination is only $42.68 \%$, which is not significantly different from the coordination in 
the No Transfer Condition. This is inconsistent with theories predicting that A would not transfer then players would coordinate (forward induction and signalling altruism).

Now consider behaviour following A choosing to transfer. The data is once again inconsistent with signalling altruism in that it predicted Low-High would be played when A transfers, but this occurs a mere $1.7 \%$ of times. Inequity-aversion and forward induction predicted High-Low, which is more consistent with the data. Following a history of A transferring High-Low is played $80.3 \%$ of the time. This constitutes $97.9 \%$ of all coordination when A transfers.

Technically, only inequity-aversion predicted Transfer-High-Low on-path. However, one step of forward induction reasoning would give the same on path prediction (see Step 1 in the explanation below Observation 3).

We had conjectured that since different motivations and reasoning processes implied different coordinated behaviour, learning would be important in the Voluntary Transfer Condition. After sufficient interaction subjects would learn about others' motivations and reasoning processes and thus be able to coordinate. Figure 12 illustrates that indeed behaviour is non-stationary over time.

Figure 12: Relative frequency of

A choosing to transfer and coordination in the Voluntary Transfer Condition by period

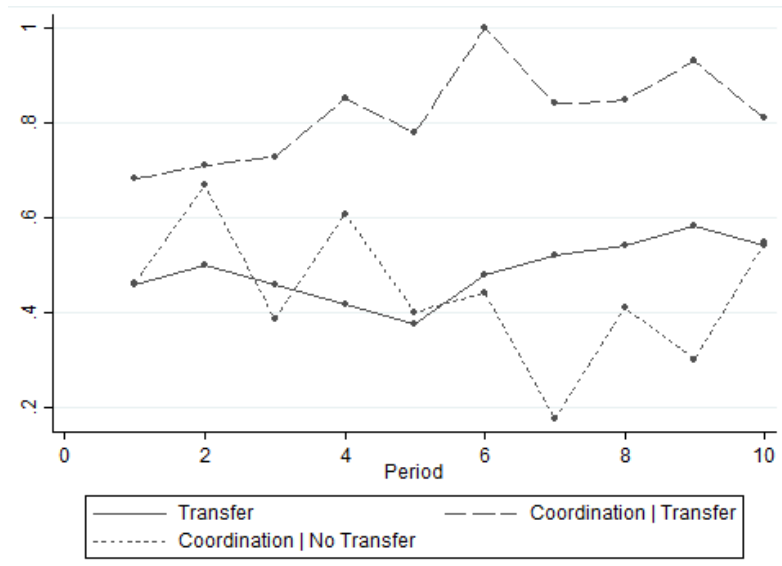

Notes: This figure depicts the relative frequency of transferring and coordination in the Voluntary Transfer Condition by period. "Transfer" represents the share of times A chose to transfer. "Coordination | No transfer" represents the share of times players coordinate following A choosing not to transfer. "Coordination | Transfer" is defined analogously.

Notice that the coordination rates conditional on each of A's transfer choices diverge over time. Since transferring leads to increasingly higher coordination, it is not surprising that A transfers more frequently over time. To better understand why the coordination rates diverge, consider second stage choices over time. 
Figure 13: Relative frequency of second-stage choices in the Voluntary Transfer Condition by period

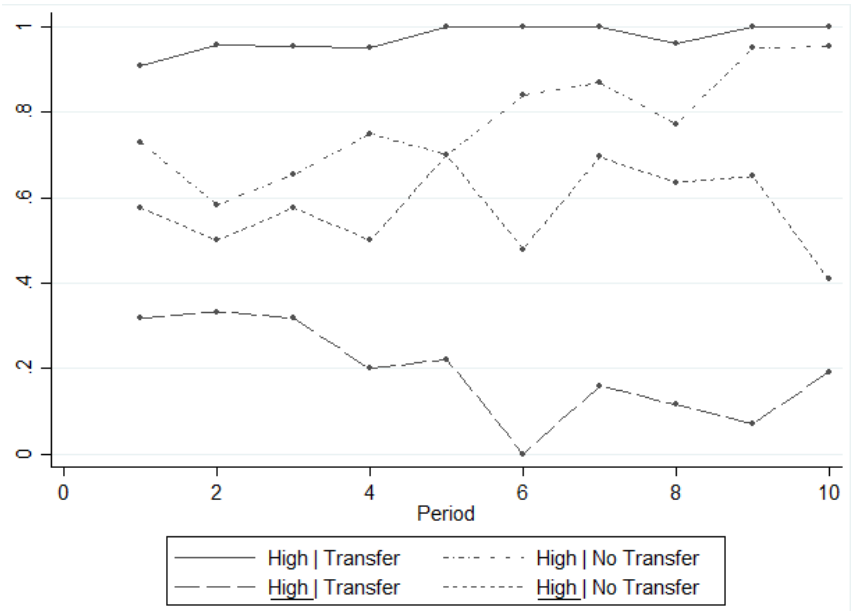

Notes: This figure depicts the share of times each second-stage choice is made in the Voluntary Transfer Condition by period. Each line depicts the share of time the named action is picked conditional on the first-stage choice. E.g. "High | Transfer" represents the share of times player B

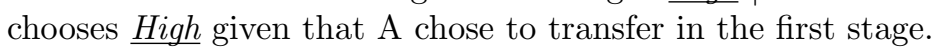

Note that after choosing Transfer, A almost always chooses High. Over time B learns this and thus chooses Low increasingly often as the session proceeds. ${ }^{14}$ Thus conditional on A having chosen Transfer, coordination increases over time.

By contrast, notice that there is little improvement in coordination when A chooses No transfer. While A chooses High more often over time, B does not choose Low any more frequently, implying persistent miscoordination.

Given the above evidence of learning, it is useful to conduct the analysis separately for earlier and later periods. Table B3 in Appendix B details the relative frequency of choices and outcomes in periods 8-10. The qualitative features discussed in relation to Table 2 are even stronger in later periods.

Result 5. In the Voluntary Transfer Condition:

a. Transfer is chosen (45.8\%/55.6\%) of the time in periods (1-7/8-10),

b. The coordination rate is (45.0\%/42.5\%) after $A$ does not transfer and (79.9\%/86.3\%) after $A$ does transfer in periods (1-7/8-10);

c. Of all coordination following A choosing to transfer, (96.7\%/100\%) is on High-Low in periods (1-7/8-10).

To summarise, Table 3 describes the share of outcomes that each theory predicts correctly.

\footnotetext{
${ }^{14}$ The relatively slow learning speed may be due to the stranger matching design.
} 
Table 3: Percentage of outcomes of the Voluntary

Transfer Condition consistent with predictions

\begin{tabular}{ccccccc}
\hline \hline & \multicolumn{3}{c}{ Path of play } & \multicolumn{3}{c}{ Including off path } \\
Periods & All & $1-7$ & $8-10$ & All & $1-7$ & $8-10$ \\
\hline Inequity-aversion & 39.17 & 35.41 & 47.92 & 68.54 & 65.18 & 73.61 \\
Forward induction & 16.45 & 16.37 & 16.67 & 55.62 & 51.78 & 64.58 \\
Signalling altruism & 17.29 & 17.56 & 16.67 & 17.29 & 17.56 & 16.67 \\
\hline
\end{tabular}

Notes: This table displays the percentage of times outcomes consistent with theoretical predictions were played in all periods, in periods 1-7 and 8-10. "Path of play" refers to the relative frequency that the path of play was predicted correctly. "Including off path" refer to the relative frequency that the path of play or off path behaviour were predicted correctly. The large difference in the explanatory power of forward induction on- and off-path is due to subjects only behaving consistently with one step of forward induction.

\section{Discussion and Conclusion}

Coordination problems are a major social challenge, especially in a world where interactions with others seem to increase over time. As we interact, we accumulate information about others' past behaviours. Since we often use decision heuristics, such information may be used when making decisions in coordination situations. We examined whether past behaviours (and knowledge of such behaviour) in other situations can help equilibrium selection in current coordination problems.

The particular past behaviour we studied is voluntary redistribution and how past redistribution can act as a potential coordination device. It is important to note that we focus on past behaviour in other situations, not learning and adaptation within the coordination situation itself (e.g. Van Huyck et al. 1997). While redistribution is usually justified on normative grounds centred on equity principles, our analysis suggests a positive justification, that it can improve coordination (cf. Bardhan 1996; Bowles and Gintis 1998).

To explore this mechanism, first we theoretically examined the effect of voluntary exante redistribution on coordination in a battle of the sexes game and identified two broad reasons why it can improve coordination. Then we tested our predictions experimentally.

The two broad reasons why voluntary ex-ante redistribution can improve coordination are as follows. First, redistributing before the coordination situation implies that one equilibrium gives a more equitable payoff distribution than the other. This facilitates coordination for players with distributive preferences (e.g. Fehr and Schmidt 2006). Second, the possibility that a player can choose whether or not to transfer, implies that her choice can signal her intended behaviour in the coordination game, thereby easing coordination. Theories of forward induction (e.g. Ben-Porath and Dekel 1992) and signalling altruism (e.g. Levine 1998, Benabou and Tirole 2006) imply coordination via such reasoning. 
To understand the relative importance of these explanations, we also analysed an intermediate game with mandatory redistribution. The key difference is that redistribution before the coordination game is mandatory for player A. As player A has to redistribute before the coordination game, forward induction and signalling are impossible candidates for explaining behaviour in the Mandatory Transfer Game, while they are possible candidates if the transfer is voluntary.

Using three experimental conditions, our experimental design allows us to disentangle the effect of distributive preferences from forward induction and signalling on coordination. By comparing coordination rates in the standard battle of the sexes to the Mandatory Transfer Game, we isolated the effect of distributive preferences. By comparing coordination rates between the Mandatory Transfer Game and the Voluntary Transfer Game conditional on a transfer being made, we isolated the effect of signalling and forward induction.

Experimentally testing our predictions we found that having the option to voluntarily redistribute increased coordination to $62.7 \%$ (from $47.7 \%$ in the game with no possibilities to redistribute). Coordination in the mandatory condition was $66.4 \%$ (not significantly different from that in the voluntary condition). One might be tempted to conclude that all behaviour is then explained by distributive preferences, however this is not the case. In the Voluntary Transfer Game, player A chose to transfer $48 \%$ of the time and conditional on A transferring, the coordination rate was $82.0 \%$, significantly higher than the coordination in the Mandatory Transfer Game. The only difference between the Voluntary Transfer Game conditional on a transfer and the Mandatory Transfer Game is that a player actively chose to transfer in the former game. Thus only signalling or forward induction can explain this difference.

Analysing choice data in more detail, we found that overall behaviour was most consistent with predictions based on distributive preferences and one-step of forward induction reasoning, rather than two steps of forward induction or signalling.

The benefit of a lab experiment is of course the ability to gather precise behavioural data in a controlled environment where causality is clear. Having said this, all experiments need to be calibrated using specific figures and this raises the question of how specific our results are to the figures used in our design. To understand the implications of our study on real world behaviour, we reconsider two central questions in light of it: Can voluntary redistribution improve coordination? And if so, why does it work?

\section{Can voluntary redistribution improve coordination?}

The theoretical analysis in Section 2.1 clearly illustrated that redistribution can imply coordination. It is encouraging that our overarching hypothesis, that the possibility to choose to redistribute before a coordination situation can improve coordination, was supported by the experimental data. However, this finding is based on the analysis of particular games. Reflecting on the generalisability of our insight is useful.

One potentially important feature of the games studied is that exactly three payoff 
units were redistributed. This created an outcome (High- $\underline{L o w}$ following the redistribution) that gave a perfectly equal payoff distribution. While the possibility of perfectly equal payoffs is unlikely in the real world, progressive redistribution does typically create the possibility of a more equal distribution (e.g. donations to charity by wealthy individuals). For past redistribution to influence coordination, a different redistribution amount (precluding perfect payoff equality), would still imply that one Nash equilibrium is more equal than the other. This fact would imply that many of the explanations driving coordination in our games would still apply.

A second and related feature of our Voluntary Transfer Condition is that there was precisely one amount that A could transfer. In the real world, one would imagine that A could choose to transfer from a range of different amounts. Our simplification may not be innocuous. For instance, theoretically a second-order coordination problem may be created in that there may be many equilibria each with different amounts being transferred. It could thus be that having the option to redistribute actually worsens coordination, as now individuals have to solve the "redistribution amount" coordination problem too.

While we cannot provide a definitive answer on how having one redistribution amount limits the generalisability of our results, we identify two reasons limiting the extent of the problem. First, even if A had a range of potential transfer amounts, within some range (i.e. neither very low nor very high), it is not clear that B would distinguish A's behaviour. This is especially true in the real world where the coordination situation is often played some time after the transfer is made, thus B may not remember the exact amount. Second, institution designers have the scope to potentially constrain the set of feasible transfers. If redistribution with a large range of voluntary transfers worsens coordination, they should simply limit the set of possible options.

A third feature of our redistribution environment relates to the asymmetry in the the game form. Only player A has the option to transfer. This asymmetry created a difference among the equilibria, thereby facilitating coordination. Suppose that both players A and $\mathrm{B}$ had the option to transfer. The game would once again be perfectly symmetric, leaving the coordination problem fully intact. Theoretically this clearly limits the usefulness of redistribution in helping coordination.

In the real world however, it may not be so problematic since individuals are rarely perfectly symmetric. Asymmetric family and work burdens imply that even if both A and B had the option to transfer, it is highly unlikely that the sizes of the transfers would be identical. The coordinating forces we have identified would then presumably apply.

Finally, it worth noting that coordination in the Voluntary Transfer Condition relied on the sequentiality of play. In situations where the transfer choice is made concurrently or after the coordination game, or too early in advance, there are much weaker reasons, if any, for why it would help coordination.

\section{Why does voluntary redistribution help coordination?}

Our experiment provided direct evidence that redistribution can improve coordination. 
We cannot of course provide such direct evidence on why it works. Our design does however allow us to discriminate between some potential explanations of why it works. Nonetheless, some reflection on the potential explanations leads one to be cautious when inferring individuals' motivations.

First, our theoretical analysis intentionally focused on motivations that could lead to a unique outcome i.e. predicted coordinated behaviour. While the motivations we studied are not exhaustive, that they are well defined and make unique predictions makes them falsifiable. Furthermore, some of them explain a large share of behaviour e.g. inequityaversion explains $73 \%$ of choices in periods $8-10$ of the Voluntary Transfer Condition. ${ }^{15}$

Second, while we believe that our analyses take the distinctive coordinating forces within each motivation to their natural conclusion, the conditions required for coordination are noteworthy. For example, our unique equilibrium with inequity-aversion relied on $\mathrm{B}$ caring more than twice as much about advantageous payoff differences than his own material payoff. ${ }^{16}$ If individuals do not have such an extreme level of inequity-aversion then the theory may be less consistent with the data than it seems.

Despite such limitations, more general insights can be extracted by comparing to previous literature. For example, Huck and Müller (2005) have shown that subjects behave consistently with two-steps of forward induction reasoning in closely related money-burning games. That the same is not true for our redistribution context (despite the theoretical reasoning applying immediately) is an important finding in itself. Redistribution does not appear to trigger the same motivations and reasoning processes as money-burning.

Third, many of our predictions were based on equilibrium reasoning. Subjects in our experiment played their condition game 30 times (ten periods, three subperiods in each, where they took on each of the three player roles in a random order). Whether or not this is long enough for convergence to equilibrium is an empirical question. There is mixed evidence of stationarity in behaviour towards the end of the game. ${ }^{17}$ Data on beliefs would be needed to better assess whether subjects are playing equilibria. However, belief elicitation is plagued with difficulties. Eliciting beliefs during the game could potentially influence behaviour in the game, eliciting them after could mean that subjects report beliefs that rationalise their behaviour ex-post.

Overall, our experiment has been useful in providing support to the theoretical idea that voluntary redistribution can improve coordination. As this discussion has illustrated, understanding the reasons why is a non-trivial endeavour, but one for which at least a first-step has now been taken towards. We leave it to future research to examine the robustness of our route to coordination and the reasons as to why it works.

\footnotetext{
${ }^{15}$ We have also identified the implications of focal point reasoning (Schelling 1960; Crawford et al. 2008; Isoni et al. 2014), indirect reciprocity (cf. Rabin 1993; Dufwenberg and Kirchsteiger 2004), moral licensing (Merritt et al 2010; Brañas-Garza et al. 2013) and team-reasoning (Bacharach 1999; Sugden $2015)$ in our games. Full details available on request.

${ }^{16}$ Note condition $\beta_{B}>2+\frac{\alpha_{B}}{2}$ in Observations 2 and 5 .

${ }^{17}$ See Figures 6, 12 and 13 .
} 


\section{References}

[1] Bacharach, M., (1999) "Interactive team reasoning: A contribution to the theory of cooperation," Research in Economics, 53(2), pp. 117-147.

[2] Bardhan, P., (1996) "Efficiency, equity and poverty alleviation: Policy issues in less developed countries," Economic Journal, 106(438), pp. 1344-1356.

[3] Ben-Porath, E. and E. Dekel, (1992) "Signaling future actions and the potential for sacrifice," Journal of Economic Theory, 57, pp. 36-51.

[4] Benabou, R. and J. Tirole, (2006) "Incentives and prosocial behavior," American Economic Review, 96(5), pp. 1652-1678.

[5] Bett, Z., Poulsen, A. and O. Poulsen (2016) "The focality of dominated compromises in tacit coordination situations: Experimental evidence," Journal of Behavioral and Experimental Economics, 60, pp. 29-34.

[6] Bolton, G. E. and A. Ockenfels (2000) "ERC: A theory of equity, reciprocity and competition," American Economic Review, 90(1), pp. 166-193.

[7] Bowles, S. and H. Gintis (1998) "Effective redistribution: New rules of markets, states and communities." In Bowles, S. and H. Gintis (Eds.) Recasting egalitarianism: New rules of communities, states and markets. Verso: London

[8] Brañas-Garza, P., Bucheli, M., Espinosa, M. P. and T. García-Muñoz (2013) "Moral cleaning and and moral licenses: Experimental evidence ," Economics and Philosophy, 29, pp. 199-212.

[9] Charness, G., Frechette, G. R. and C.-Z. Qin (2007) "Endogenous transfers in the prisoner's dilemma game: An experimental test of cooperation and coordination," Games and Economic Behavior, 60(2), pp. 287-306.

[10] Charness, G. and M. Rabin (2002) "Understanding social preferences with simple tests," Quarterly Journal of Economics, 117(3), pp. 817-869.

[11] Chen, R. and Y. Chen (2011). "The potential of social identity for equilibrium selection," American Economic Review, 101(6), pp. 2562-2589.

[12] Cooper, R., DeJong, D. V., Forsythe, R. and T. W. Ross (1989) "Communication in the battle of the sexes: Some experimental results," RAND Journal of Economics, 20(4), pp. $568-587$.

[13] Cooper, R., DeJong, D. V., Forsythe, R. and T. W. Ross (1993) "Forward induction in the battle-of-the-sexes games," American Economic Review, 83(5), pp. 1303-1316.

[14] Crawford, V. P., Gneezy, U. and Y. Rottenstreich (2008) "The power of focal points is limited: Even minute payoff asymmetry may yield large coordination failure," American Economic Review, 98(4), pp. 1443-1458. 
[15] van Damme, E. (1989) "Stable equilibria and forward induction," Journal of Economic Theory, 48(2), pp. 476-496.

[16] Dufwenberg, M. and G. Kirchsteiger (2004) "A theory of sequential reciprocity," Games and Economic Behavior, 47, pp. 268-298.

[17] Dufwenberg, M. and A. Patel (2017) "Reciprocity networks and the participation problem," Games and Economic Behavior, 101, pp. 260-272.

[18] Falk, A. and J. Heckman (2009) "Lab experiments are a major source of knowledge in the social sciences," Science, 326(530), pp. 535-538.

[19] Fehr, E. and K. M. Schmidt (1999) "A theory of fairness, competition and cooperation," Quarterly Journal of Economics, 114(3), pp. 817-868.

[20] Fehr, E. and K. M. Schmidt (2006) "The economics of fairness, reciprocity and altruism - Experimental evidence." In Kolm, S. and J. M. Ythier (Eds.) Handbook of the Economics of Giving, Altruism and Reciprocity. Volume 1. Elsevier: New York

[21] Fischbacher, U. (2007) "Z-tree: Zurich toolbox for ready-made economic experiments," Experimental Economics, 10(2), pp. 171-178.

[22] Greiner, B. (2015) "Subject pool recruitment procedures: Organizing experiments with ORSEE," Journal of the Economic Science Association, 1(1), pp. 114-125.

[23] Huck, S. and W. Müller (2005) "Burning money and (pseudo) first-mover advantages: An experimental study on forward induction," Games and Economic Behavior, 51(1), pp. 109-127.

[24] Isoni, A., Poulsen, A., Sugden, R. and K. Tsutsui (2014) "Efficiency, equality and labelling: An experimental investigation of focal points in explicit bargaining," American Economic Review, 104(10), pp. 3256-3287.

[25] Jackson. M. O. and S. Wilkie (2005) "Endogenous games and mechanisms: Side payments among players," Review of Economic Studies, 72(2), pp. 543-566.

[26] Kohlberg, M. and J.-F. Mertens (1986) "On the strategic stability of equilibria," Econometrica, 54(5), pp. 1003-1037.

[27] Levine, D. K. (1998) "Modelling altruism and spitefulness in experiments," Review of Economic Dynamics, 1, pp. 593-622.

[28] López-Pérez, R., Pintér, Á. and H. J. Kiss (2015) "Does payoff equality facilitate coordination? A test of Schelling's conjecture," Journal of Economic Behavior and Organization, 117, pp. 209-222.

[29] Merritt, A. C., Effron, D. and B. Monin (2010) "Moral self-licensing: when being good frees us to be bad," Social and Personality Psychology Compass, 4/5, pp. 344-357.

[30] Rabin, M. (1993) "Incorporating fairness into game theory and economics," American Economic Review, 83(5), pp. 1281-1302. 
[31] Schelling, T. C. (1960) The Strategy of Conflict. Harvard University Press. Cambridge: MA.

[32] Shahriar, Q. (2013) "Forward induction and other regarding preferences arising from an outside option: An experimental investigation," Journal of Management and Strategy, $4(4)$, pp. $52-57$.

[33] Sugden, R. (2015) "Team reasoning and intentional cooperation for mutual benefit," Journal of Social Ontology, 1(1), pp. 143-166.

[34] Van Huyck, J. B., Gillette, A.B. and R. C. Battalio (1992) "Credible assignments in coordination games," Games and Economic Behavior, 4(4), pp. 606-626.

\section{Appendix}

\section{A. Proofs of Observations}

Proof of Observation 2 (Inequity aversion in $\Gamma_{V}$ ) Let $\alpha_{A}+\beta_{A} \geq 2$ and $\beta_{B}>2+\frac{\alpha_{B}}{2}$ (note that since $\beta_{B} \leq \alpha_{B}, \beta_{B}>2+\frac{\alpha_{B}}{2}$ implies that $\alpha_{B}>2+\frac{\alpha_{B}}{2} \Rightarrow \alpha_{B}>4$ ). To find SPE, solve backwards.

First consider the subgame following $N$. Suppose $H \underline{H}$ is played following $N$. A does not deviate to $L$ if $U_{A}(H \underline{H} \mid N) \geq U_{A}(L \underline{H} \mid N) \Rightarrow 0 \geq 3-\frac{\alpha_{A}}{2}(6-3)-\frac{\beta_{A}}{2}(3-0) \Rightarrow$ $\alpha_{A}+\beta_{A} \geq 2$, which is true. B does not deviate to $l$ if $U_{B}(H \underline{H} \mid N) \geq U_{B}(H \underline{L} \mid N) \Rightarrow$ $0 \geq 3-\frac{\alpha_{B}}{2}(6-3)-\frac{\beta_{B}}{2}(3-0) \Rightarrow \alpha_{B}+\beta_{B} \geq 2$, which is true. Thus $H \underline{H}$ is a NE of this subgame. Now suppose $H \underline{L}$ is played following $N$. B deviates to $h$ if $U_{B}(H \underline{H} \mid N)>$ $U_{B}(H \underline{L} \mid N) \Rightarrow 0>3-\frac{\alpha_{B}}{2}(6-3)-\frac{\beta_{B}}{2}(3-0) \Rightarrow \alpha_{A}+\beta_{A}>2$, which is true. Thus $H \underline{L}$ is not a NE of this subgame. Now suppose $L \underline{H}$ is played. B deviates to $l$ if $U_{B}(L \underline{L} \mid N)>$ $U_{B}(L \underline{H} \mid N) \Rightarrow 0>6-\frac{\beta_{B}}{2}(6-3+6-0) \Rightarrow \beta_{B}>\frac{4}{3}$, which is true. Thus $L \underline{H}$ is not a NE of this subgame. Finally suppose $L \underline{L}$ is played. A does not deviate to $H$ if $U_{A}(L \underline{L} \mid N) \geq U_{A}(H \underline{L} \mid N) \Rightarrow 0 \geq 6-\frac{\beta_{A}}{2}(6-3+6-0) \Rightarrow \beta_{A} \geq \frac{4}{3}$, but does otherwise. B does not deviate to $h$ if $U_{B}(L \underline{L} \mid N) \geq U_{B}(L \underline{H} \mid N) \Rightarrow 0 \geq 6-\frac{\beta_{B}}{2}(6-3+6-0) \Rightarrow \beta_{B} \geq \frac{4}{3}$, which is true. Thus $L \underline{L}$ is a NE of this subgame if $\beta_{A} \geq \frac{4}{3}$.

Now consider the subgame following $T$. Suppose $H \underline{H}$ is played following $T$. A deviates to $L$ if $U_{A}(L \underline{H})>U_{A}(H \underline{H}) \Rightarrow 0-\frac{\alpha_{A}}{2}(6-0+3-0)>-3-\frac{\alpha_{A}}{2}(3--3+0--3) \Rightarrow 0>-3$, which is true. Thus $H \underline{H}$ is not a NE. Now Suppose $H \underline{L}$ is played following $T$. A does not deviate to $L$ if $U_{A}(H \underline{L}) \geq U_{A}(L \underline{L}) \Rightarrow 3 \geq-3-\frac{\alpha_{A}}{2}(3--3+0--3) \Rightarrow \alpha_{A} \geq-\frac{4}{3}$, which is true. B does not deviate to $h$ if $U_{B}(H \underline{L}) \geq U_{B}(H \underline{H}) \Rightarrow 3 \geq 0-\frac{\alpha_{B}}{2}(3-0)-\frac{\beta_{B}}{2}(0--3) \Rightarrow$ $\alpha_{A}+\beta_{B} \geq-2$, which is true. Thus $H \underline{L}$ is a NE. Now suppose $L \underline{H}$ is played following $T$. B deviates to $l$ if $U_{B}(L \underline{L})>U_{B}(L \underline{H}) \Rightarrow 0-\frac{\alpha_{B}}{2}(3-0)-\frac{\beta_{B}}{2}(0--3)>6-\frac{\beta_{B}}{2}(6-3+6-0) \Rightarrow$ $\beta_{B}>2+\frac{\alpha_{B}}{2}$, which is true. Thus $L \underline{H}$ is not a NE. Now suppose $L \underline{L}$ is played following $T$. A deviates to $H$ if $U_{A}(H \underline{L})>U_{A}(L \underline{L}) \Rightarrow 3>-3-\frac{\alpha_{B}}{2}(0--3+3--3) \Rightarrow \alpha_{A}>-\frac{4}{3}$, 
which is true. Thus $L \underline{L}$ is not a NE. Overall then, $H \underline{L}$ is the unique NE of the subgame following $T$.

Finally consider A's choice at the initial node given second stage NE behaviour. If $\beta_{A}<\frac{4}{3}$ then $H \underline{H}$ is played following $N$ and $H \underline{L}$ is played following $T$. Given this, A plays $N$ if $U_{A}(N H H, \underline{H L}) \geq U_{A}(T H H, \underline{H L}) \Rightarrow 0 \geq 3$, which is false, thus A chooses $T$ at the initial node. The unique SPE is thus $\{T H H, \underline{H L}\}$. If $\beta_{A} \geq \frac{4}{3}$ either $H \underline{H}$ or $L \underline{L}$ is played following $N$ and $H \underline{L}$ is played following $T$. Suppose $H \underline{H}$ is played following $N$. A plays $N$ if $U_{A}(N H H, \underline{H L}) \geq U_{A}(T H H, \underline{H L}) \Rightarrow 0 \geq 3$, which is false, thus A plays $T$. Suppose $L \underline{L}$ is played following $N$. A plays $N$ if $U_{A}(N L H, \underline{L L}) \geq U_{A}(T L H, L L) \Rightarrow 0 \geq 3$, which is false, thus A plays $T$. Hence there are two SPE, $\{T H H, \underline{H L}\}$ and $\{T L H, \underline{L L}\}$.

Proof of Observation 3 (Forward Induction in $\Gamma_{V}$ ) $\Gamma_{V}$ falls into the class of games studied by Ben-Porath and Dekel (1992). Within this class, they demonstrate that applying Iterated Elimination of Weakly Dominated Strategies (IEWDS) selects the forward induction solution. The reduced normal form of $\Gamma_{V}$ is depicted below. ${ }^{18}$

Figure 14: Reduced normal form of $\Gamma_{V}$

\begin{tabular}{cccccc} 
& & \multicolumn{4}{c}{ Player B } \\
& & $\underline{H H}$ & $\underline{H L}$ & $\underline{L H}$ & $\underline{L L}$ \\
\cline { 3 - 6 } Player A & 0,0 & 0,0 & 6,3 & 6,3 \\
& $N L \cdot$ & 3,6 & 3,6 & 0,0 & 0,0 \\
& $T \cdot H$ & $-3,0$ & 3,3 & $-3,0$ & 3,3 \\
& $T \cdot L$ & 0,6 & $-3,0$ & 0,6 & $-3,0$ \\
\hline
\end{tabular}

Apply IEWDS as follows:

Step 1: Delete $T \cdot L$ (weakly dominated by $N H \cdot$ ).

Step 2: Delete $\underline{H H}$ (weakly dominated by $\underline{H L}$ in the residual game).

Step 3: Delete $\underline{L H}$ (weakly dominated by $\underline{L L}$ in the residual game).

Step 4: Delete $N L$. (weakly dominated by $T \cdot H$ in the residual game).

Step 5: Delete $\underline{H L}$ (weakly dominated by $\underline{L L}$ in the residual game).

Step 6: Delete $T \cdot H$ (weakly dominated by $N H$. in the residual game).

This leaves $\{N H \cdot, \underline{L L}\}$ as the forward induction solution.

Proof of Observation 4 (Signalling altruism in $\Gamma_{V}$ ) Note that in any PBE it must be that players play the NE profiles in the final stage (i.e. $H \underline{L}$ or $L \underline{H}$ ). If not, both A and $\mathrm{B}$ would have an incentive to deviate in the final stage as unilateral deviation strictly increases own and the other players' payoffs.

\footnotetext{
${ }^{18}$ Note that $N H \cdot=\{N H H, N H L\}, N L \cdot=\{N L H, N L L\}, T \cdot H=\{T H H, T L H\}$ and $T \cdot L=$ $\{T H L, T L L\}$.
} 
In a separating PBE, types $g$ and $m$ must make different choices in the transfer stage. Consider a candidate equilibrium where a $g$ type plays $N$ and a $m$ type plays $T$. If the same action profile is played in the final stage, or $H \underline{L}$ is played following $N$ and $L \underline{H}$ is played following $T$, then $m$ would deviate to $N$ as doing so can strictly increase her material payoff. Thus it must be that in this candidate equilibrium, $H \underline{L}$ is played following $T$ and $L \underline{H}$ is played following $N$. However, if that were the case, then a $g$ type will deviate to $T$ if $3+\alpha_{g}(0+6)<6+\alpha_{g}(3+3) \Rightarrow 3<6$, which is true. Thus there exist no PBE where $g$ plays $N$ and $m$ plays $T$.

Now consider candidate equilibria where $g$ plays $T$ and $m$ plays $N$. If the same action profile is played in the final stage for all histories, then deviation in stage 1 by a $m$ type A does not strictly reduce A's payoff, thus this cannot be part of a sequentially strict PBE. If $H \underline{L}$ is played after $T$ and $L \underline{H}$ is played after $N$, then a $m$ type can increase her material payoff by deviating to $T$, thus this cannot be part of a PBE. The only remaining possibility is that $L \underline{H}$ is played after $T$ and $H \underline{L}$ is played after $N$. A $m$ type has no incentive to deviate to $T$ as doing so would reduce her material payoff. A $g$ type does not deviate to $N$ if $0+\alpha_{g}(6+3)>6+\alpha_{g}(3+0) \Rightarrow \alpha_{g}>1$. Thus this is a PBE.

Proof of Observation 5 (Inequity-aversion in $\Gamma_{M}$ ) See proof of Observation 2, in the subgame following $T$.

\section{B. Additional data analysis}

This appendix contains additional data analysis that is not directly relevant for testing our hypotheses.

\section{No Transfer Condition Versus Mandatory Transfer Condition}

The coordination rate in the No Transfer Condition is $47.7 \%$, whereas that in the Mandatory Transfer Condition is $66.4 \%$, a statistically significant difference (Fisher's Exact test, $p<0.001$ ). The figure below depicts the dynamic statistical significance in the difference in coordination between the two treatments. 
Figure B1: The effect of a mandatory transfer on coordination

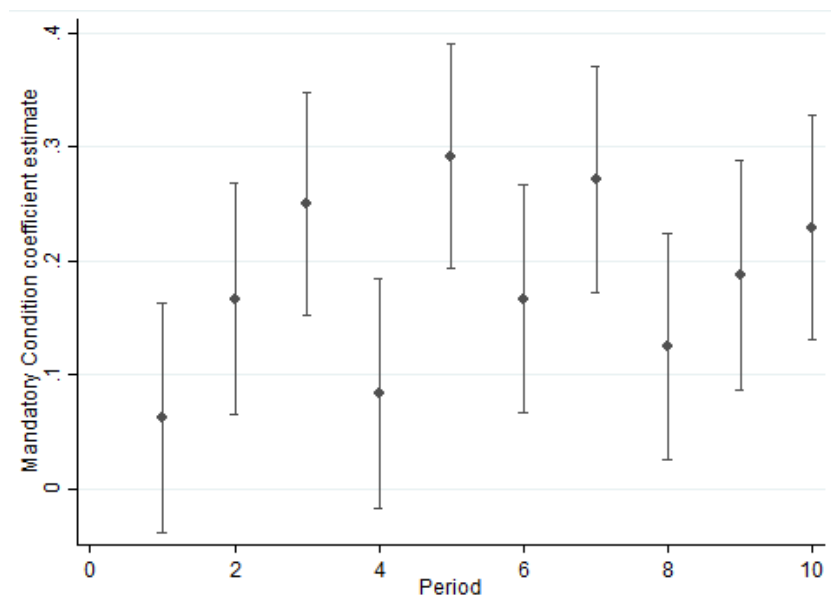

Notes: This figure displays treatment effect estimates and $95 \%$ confidence intervals from periodwise OLS regressions where the dependent variable is Coordination (a dummy for whether or not the group coordinated). Data from the No Transfer and Mandatory Transfer Conditions were pooled, the treatment is a dummy variable for the Mandatory Transfer Condition. Controls for the subperiod were included and robust standard errors clustered at session level were used.

The difference in coordination is statistically significant in albeit two periods. ${ }^{11}$

\section{Behaviour in the No Transfer Condition}

The table below shows the relative frequency of choices and outcomes in the No Transfer Condition. The coordination rate is $47.70 \%$ which is not significantly different from the mixed strategy NE prediction of $44.4 \%$ coordination rate (a test of proportions (PR), does not reject the null that the coordination rate equals $44.4 \%, p=0.101$ ).

Table B1: Relative frequency of choices and outcomes in the No Transfer Condition

\begin{tabular}{lccccc}
\hline \hline 480 obs. & \multicolumn{4}{c}{ Player B } & \\
& & $\underline{\text { High }}$ & $\underline{\text { Low }}$ & \\
\multirow{2}{*}{ Player A } & High & 40.42 & 32.5 & 72.92 \\
& Low & 15.2 & 11.88 & 27.08
\end{tabular}

$55.62 \quad 44.38$

Notes: This table displays the percentage of times each outcome was played in the No Transfer Condition. The percentage of times a particular action was played is stated on the right and below the main table. Coordinated outcomes are shaded. 
Notice that player A subjects play High $72.9 \%$ of the time, while B plays $\underline{\text { High only }}$ $55.6 \%$ of the time, both proportions are significantly different from the predicted $\frac{2}{3}$ probability of playing High or High (PR tests, $p=0.004$ and $p<0.001$ respectively). This difference between $\mathrm{A}$ and $\mathrm{B}$ roles fits with previous literature finding that labels and pseudo-first moves (e.g. Huck and Müller 2005; Isoni et al. 2014) aid coordination.

As such effects are present in the Voluntary Transfer Condition, the No Transfer Condition is an important control to separate the effect of the redistribution from such experimental confounds.

\title{
Behaviour in the Mandatory Transfer Condition
}

The next table states the relative frequency of choices and outcomes in the Mandatory Transfer Condition. When subjects coordinate, $96.2 \%$ of the time it is on High-Low.

Table B2: Relative frequency of choices and outcomes in the Mandatory Transfer Condition

\begin{tabular}{ccc|c|c|}
\hline \hline 480 obs. & & \multicolumn{4}{c}{ Player B } & \\
& & $\underline{\text { High }}$ & $\underline{\text { Low }}$ & \\
Player A & High & 23.33 & 63.54 & 86.87 \\
& Low & 2.5 & 10.62 & 13.12
\end{tabular}

$25.83 \quad 74.16$

\begin{abstract}
Notes: The table displays the percentage of times each outcome was played in the Mandatory Transfer Condition. The percentage of times a particular action was played is stated on the right and below the main table. Coordinated outcomes are shaded.
\end{abstract}

\section{Behaviour in the Voluntary Transfer Institution periods 8-10}

The following table states the relative frequency of choices and outcomes in the Voluntary Transfer Condition in periods 8-10. 
Table B3: Relative frequency of choices and outcomes in the Voluntary Transfer Condition in periods 8-10

\begin{tabular}{|c|c|c|c|c|}
\hline \multicolumn{5}{|c|}{ Panel A: Unconditional } \\
\hline \multirow{4}{*}{$\begin{array}{l}144 \text { obs. } \\
\text { Player A }\end{array}$} & \multicolumn{4}{|c|}{ Player B } \\
\hline & \multirow{3}{*}{$\begin{array}{l}\text { High } \\
\text { Low }\end{array}$} & $\underline{\text { High }}$ & $\underline{L o w}$ & \multirow{3}{*}{$\begin{array}{c}94.44 \\
5.56\end{array}$} \\
\hline & & 29.86 & 64.58 & \\
\hline & & 2.08 & 3.47 & \\
\hline & \multicolumn{4}{|c|}{$31.94 \quad 68.06$} \\
\hline \multicolumn{5}{|c|}{ Panel B: Following No Transfer } \\
\hline \multirow[t]{2}{*}{64 obs. } & \multicolumn{4}{|c|}{ Player B } \\
\hline & & High & $\underline{\text { Low }}$ & \\
\hline \multirow{3}{*}{ Player A } & High & $\overline{51.56}$ & $\overline{37.50}$ & 89.06 \\
\hline & Low & 4.69 & 6.25 & 10.94 \\
\hline & & 56.25 & 43.75 & \\
\hline \multicolumn{5}{|c|}{ Panel C: Following Transfer } \\
\hline \multirow[t]{2}{*}{80 obs. } & \multicolumn{4}{|c|}{ Player B } \\
\hline & \multirow{3}{*}{$\begin{array}{l}\text { High } \\
\text { Low }\end{array}$} & $\underline{\text { High }}$ & $\underline{\text { Low }}$ & \multirow{3}{*}{$\begin{array}{c}98.75 \\
1.25\end{array}$} \\
\hline \multirow{3}{*}{ Player A } & & $\overline{12.5}$ & $\overline{86.25}$ & \\
\hline & & 0 & 1.25 & \\
\hline & & 12.5 & 87.5 & \\
\hline
\end{tabular}

Notes: This table displays the percentage of times each secondstage outcome was played in periods 8-10 of the Voluntary Transfer Condition: overall (Panel A), following No transfer (Panel B) and following Transfer (Panel C). The percentage of times a particular action was played is stated on the right and below each table. Coordinated outcomes are shaded.

\section{Instructions}




\section{Welcome!}

You are now taking part in an economic experiment which has been financed by various science foundations. Please read the instructions carefully before the experiment starts.

These instructions are solely for your own information. Do not communicate with other people in this room during the course of the session. Should you have any questions please ask us by raising your hand and you'll be answered privately.

If you violate this rule, you shall be excluded from the experiment and from all payments.

You will receive a show-up fee of 4 Euros for being here, in addition to your earnings from the experiment.

The experiment is divided into two parts.

These instructions refer to Part I. The instructions for Part II will be distributed at the end of Part I.

During the experiment we shall not speak of Euros but rather of Points: your entire earnings will be computed in Points. At the end of the experiment the total amount of Points you have earned in each Part will be converted to Euros at predefined exchange rates.

Notice: All parts are independent of each other and no behavior or earnings in one part affects behavior and earnings in another part.

Your earnings from the experiment will be given by the sum of your earnings in the two parts.

You will be told your final earnings, including the show-up fee, at the end of the experiment. It will be paid in cash.

The experiment is computerized; hence all your choices and actions will be made via the computer terminal in front of you. Similarly, all the information you receive during the course of the experiment will be given on the computer screen.

Every choice you make will be recorded in data files and linked to each other via an anonymous identification number. Therefore we will never be able to link specific choices to the identity of the person taking them.

Also no other person in this room will ever know which choices you made, nor will you be informed of which person in the room made a particular choice.

The information on the following pages describes the experiment in detail.

Should you have any questions, please raise your hand.

NB: Please return all materials at the end of the experiment! 


\section{Part I}

We will first describe an interaction situation between three individuals. Once we have described the situation we will inform you of your task.

\section{Description of the interaction situation}

Imagine a group of three people. We will refer to them as the Participants of the situation and name them Participant A, Participant B, and Participant C. Each of them has 12 Points in their own account at the beginning of the interaction, and each will walk away with an amount of Points determined by their choices during the interaction. Their final Points can be greater, smaller than or equal to the initial 12 Points. The interaction situation proceeds in two stages between Participants $A$ and $C$. Participant B is a bystander and never makes a choice.

\section{Stage 1}

Participants enter Stage 1 with the 12 Points they have in their accounts.

In Stage 1, Participant A can transfer 3 points from his/her account to that of Participant B. Therefore participant $A$ will choose one of the two options below:

\section{Option 1: Transfer 3 Points to Participant B}

Option 2: Don't Transfer 3 Points to Participant B

After Participant A has made his or her choice, all Participants in the group are informed about the decision and the number of points currently in their accounts.

\section{Stage 2}

Participants enter Stage 2 with the number of Points they had in their accounts at the end of Stage 1. In Stage 2, Participants $A$ and $C$ simultaneously choose between two alternatives on how much to increase their points by:

\section{Alternative 1: 6 Points for Participant A and 3 Points for Participant C}

\section{Alternative 2: 3 Points for Participant A and 6 Points for Participant C}

If they simultaneously and independently choose the same alternative, then the number of points indicated in the chosen alternative will be added to the Points they have in their accounts. If not, they get zero additional points.

After Participants $\mathrm{A}$ and $\mathrm{C}$ have chosen, all Participants are told the outcome and the number of points in their accounts. The interaction then ends. 


\section{Your task}

As soon as the experiment starts you will be given additional detailed information on screen. Make sure to read that information carefully.

When the experiment starts, at the beginning of Round 1 of Period 1 , you will randomly be given one of the roles, Participant A, B, or C, and will randomly be assigned to a group of 3 . You will then take part in the interaction in the Participant role you've been given with the group you've been assigned to (an example will be provided in the following pages).

After the interaction is over (that is, after Participants $A$ and $C$ made their Stage 2 choices), your group will be broken up and Round 2 of Period 1 will start.

In Round 2 of Period 1 you will randomly be given a new role (one of the two roles you were not assigned in Round 1: if, for example, you were assigned the role of Participant $A$ in Round 1, in Round 2 you will be randomly assigned to the role of Participant B or Participant $C$ ) and randomly assigned to a new group of 3 . You then take part in the interaction in the new Participant role and new group you were given. After the interaction is over, your group will again be broken up and Round 3 will start.

In Round 3 of Period 1 you will be given the role you have not yet been assigned (continuing with the previous example, if you were Participant $A$ in Round 1 and Participant $C$ in Round 2 then in Round 3 you will be Participant $B$ ), you will be assigned to a new group of 3 and take part in the interaction again. After the interaction is over, your group will once again be broken up.

After Round 3 is over you move on to Period 2, you will receive a new group and a new role, and the steps are repeated. This will be repeated for 10 Periods. After the $10^{\text {th }}$ Period Part I will end and there will be no more Rounds or Periods.

Notice that in each Round within a Period you will be given a different role, and that the group you are assigned to in each Round is always randomly formed throughout the experiment. 
Diagram 1: Randomization in Role and Group

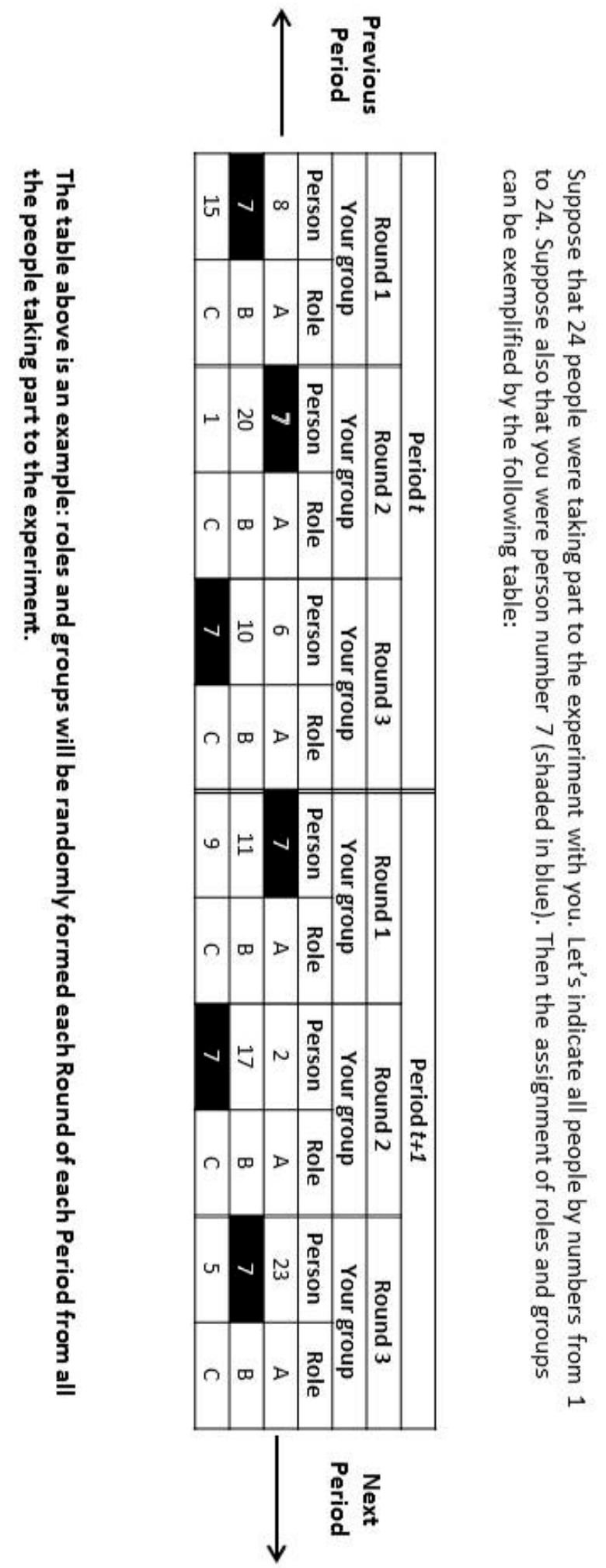




\section{Your earnings from Part I}

Your earnings from one specific Round will be given by the Points accumulated in your Participant's account under the role you've taken part in during that specific Round. At the end of Part I, the software will randomly draw two numbers: one number between 1 and 10, corresponding to one of the Periods, and one number between 1 and 3, corresponding to one of the three Rounds of a Period. Your earnings from Part I of the experiment will be determined by the number of Points in your account in the Period and Round corresponding to the two numbers extracted, and will be converted into Euros at the following exchange rate: 1 Point $=0.5$ Euros.

Find this illustrated in Diagram 2.

Since all choices have the same probability of being relevant for payoff, it is in your best interest to make each choice as if it were the choice that counts. 
Diagram 2: Period and Round structure, and Earnings selection

\begin{tabular}{|c|c|c|c|}
\hline \multicolumn{4}{|c|}{ Example: Periods and Rounds } \\
\hline Period & Round & $\begin{array}{l}\text { Your } \\
\text { Role }\end{array}$ & $\begin{array}{c}\text { Your } \\
\text { Earnings }\end{array}$ \\
\hline \multirow{3}{*}{1} & 1 & $\mathrm{~A}$ & \# Points \\
\hline & 2 & C & \# Points \\
\hline & 3 & B & \# Points \\
\hline \multirow{3}{*}{2} & 1 & B & \# Points \\
\hline & 2 & A & \# Points \\
\hline & 3 & C & \# Points \\
\hline \multirow{3}{*}{3} & 1 & C & \# Points \\
\hline & 2 & $B$ & \# Points \\
\hline & 3 & A & \# Points \\
\hline \multirow{3}{*}{4} & 1 & A & \# Points \\
\hline & 2 & B & \# Points \\
\hline & 3 & C & \# Points \\
\hline \multirow{3}{*}{5} & 1 & -- & \# Points \\
\hline & 2 & -- & \# Points \\
\hline & 3 & -- & \# Points \\
\hline \multirow{3}{*}{6} & 1 & -- & \# Points \\
\hline & 2 & -- & \# Points \\
\hline & 3 & -- & \# Points \\
\hline \multirow{3}{*}{7} & 1 & -- & \# Points \\
\hline & 2 & -- & \# Points \\
\hline & 3 & -- & \# Points \\
\hline \multirow{3}{*}{8} & 1 & -- & \# Points \\
\hline & 2 & -- & \# Points \\
\hline & 3 & -- & \# Points \\
\hline \multirow{3}{*}{9} & 1 & -- & \# Points \\
\hline & 2 & -- & \# Points \\
\hline & 3 & -- & \# Points \\
\hline \multirow{3}{*}{10} & 1 & -- & \# Points \\
\hline & 2 & -- & \# Points \\
\hline & 3 & -- & \# Points \\
\hline
\end{tabular}

The table to the left is an example! Roles will be assigned at random, and not necessarily in the sequence represented in the table.

To the left, you can see how the experiment is structured in Periods and Rounds: each Period is divided into 3 Rounds. In each Round you will take part to the interaction in a different Role, as in the example. In each Period you will take part in each Role once.

Suppose that at the end the numbers were extracted as follows:

\section{Period extraction}

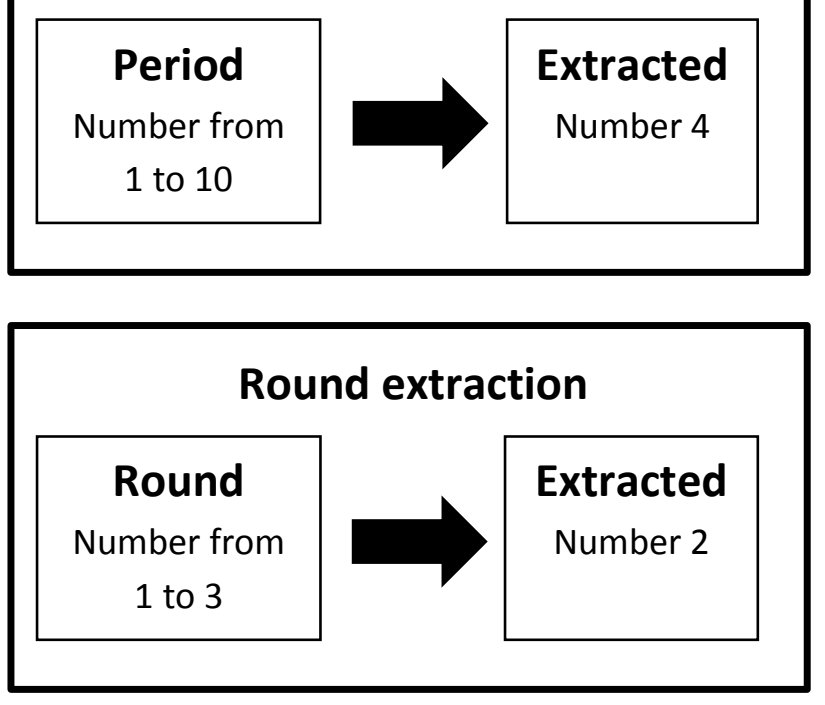

Then your earnings from Part I of the experiment will be given by the Points earned in Round 2 of Period 4 , as illustrated in the table to the left (highlighted Period and Round). 


\section{Part II}

In Part II you will make a series of allocation choices among several alternatives. As in Part I of the experiment, we will not speak of Euros, but rather Points.

The Points from Part II will be converted into euros at a rate of

20 Points = 1 Euro (or 1 Point=0.05 Euros).

You will be randomly paired with another person, whom we will refer to as the other. You will not know who the other person is, nor will the other person be informed about your identity.

You will be making a series of decisions about allocating resources between you and this other person. For each of the questions, please indicate the distribution you prefer most by selecting the corresponding button in the middle row. You can only make one choice for each question.

Your decisions will yield money for both yourself and the other person. In the example in Diagram 4 a person has chosen to distribute Points so that he/she receves 50 Points, while the anonymous other person receives 40 Points.

\section{Diagram 3: Example of an allocation choice}

In the example below, a person chose to the allocation giving 50 Points to herself, and 40 points to the unknown other person.

\begin{tabular}{|c|c|c|c|c|c|c|c|c|c|}
\hline $\begin{array}{c}\text { You } \\
\text { receive }\end{array}$ & 30 & 35 & 40 & 45 & 50 & 55 & 60 & 65 & 70 \\
\hline & 0 & 0 & 0 & 0 & $\bullet$ & 0 & 0 & 0 & 0 \\
\hline $\begin{array}{c}\text { Other } \\
\text { receives }\end{array}$ & 80 & 70 & 60 & 50 & 40 & 30 & 20 & 10 & 0 \\
\hline
\end{tabular}

In terms of Euros, this yields an allocation of 50/20=2.5 Euros for the person making the choice, and of $40 / 20=2$ Euros for the unknown other.

There are no right or wrong answers, this is all about personal preferences.

As you can see, your choices influence both the number of Points you receive, as well as the number of Points the other person receives.

After you have made all your choices, the software will randomly assign one person from your group (you or the other) the role of "Receiver" and the other the role of the "Sender". One of the allocation choices made by the Sender will be randomly selected by the software. This allocation will be paid in cash to both the Sender and the Receiver.

The No Speaking rule still applies! If you have any questions, please raise your hand.

NB: Please return all materials at the end of the experiment! 